\title{
EIGENVALUES AND EIGENVECTORS OF SEMIGROUP GENERATORS OBTAINED FROM DIAGONAL GENERATORS BY FEEDBACK*
}

\author{
CHENG-ZHONG XU ${ }^{\dagger}$ AND GEORGE WEISS ${ }^{\ddagger}$
}

\begin{abstract}
We study infinite-dimensional well-posed linear systems with output feedback such that the closed-loop system is well-posed. The generator $A$ of the open-loop system is assumed to be diagonal, i.e., the state space $X$ (a Hilbert space) has a Riesz basis consisting of eigenvectors of $A$. We investigate when the closed-loop generator $A^{K}$ is Riesz spectral, i.e, its generalized eigenvectors form a Riesz basis in $X$. We construct a new Riesz basis in $X$ using the sequence of eigenvectors of $A$ and the control operator $B$. If this new basis is, in a certain sense, close to a subset of the generalized eigenvectors of $A^{K}$, then we conclude that $A^{K}$ is Riesz spectral. This approach leads to several results on Riesz spectralness of $A^{K}$ where the closed-loop eigenvectors need not be computed. We illustrate the usefulness of our results through several examples concerning the stabilization of systems described by partial differential equations in one space dimension. For the systems in the examples we show that the closed-loop generator is Riesz spectral. Our method allows us to simplify long computations which were necessary otherwise.
\end{abstract}

Key words: strongly continuous semigroup, eigenvalues and eigenvectors, well-posed linear system, static output feedback, characteristic equation, exact controllability, Riesz basis, wave equation, beam equation.

1. Introduction. In this paper we consider a well-posed linear system $\Sigma$ with input $u$ and output $y$ connected in a feedback loop, as shown in Figure 1. If the bounded feedback operator $K$ is admissible for $\Sigma$, then the feedback connection $u=K y+v$ gives rise to a new well-posed linear system with input $v$, called the closedloop system and denoted by $\Sigma^{K}$. We refer to Weiss [42] for the concepts mentioned above. We denote by $A$ and $A^{K}$ the semigroup generators of $\Sigma$ and $\Sigma^{K}$. We denote by $\mathbf{G}$ and $\mathbf{G}^{K}$ the transfer functions of $\Sigma$ and $\Sigma^{K}$, so that

$$
\mathbf{G}^{K}=(I-\mathbf{G} K)^{-1} \mathbf{G}=\mathbf{G}(I-K \mathbf{G})^{-1} .
$$

We now introduce some terminology. Denote the state space of $\Sigma$ by $X$. The operator $A$ and the semigroup $\mathbb{T}$ on $X$ generated by $A$ are called diagonal if there is a sequence $\left(\lambda_{n}\right)$ of eigenvalues of $A$ and a corresponding sequence of eigenvectors $\left(e_{n}\right)$ (i.e., $\left.\left(\lambda_{n} I-A\right) e_{n}=0\right)$ such that $\left(e_{n}\right)$ is a Riesz basis in $X$. A nonzero $\xi \in X$ is called a generalized eigenvector of $A$ if $(\lambda I-A)^{m} \xi=0$ for some $\lambda \in \mathbb{C}$ and some

* Dedicated to John Baillieul on the Occasion of His 65 th Birthday.

${ }^{\dagger}$ Corresponding author, tel. (+33)472431890, fax. (+33)472431699. Université de Lyon, LAGEP, Bât. CPE, Université Lyon 1, 43, Boulevard du 11 Novembre 1918, 69622 Villeurbanne Cedex, France. E-mail: xu@lagep.univ-lyon1.fr; The corresponding author is also with Department of Mathematics and Information, Ludong University, Yantai, China.

${ }^{\ddagger}$ Department of Electrical Engineering - Systems, Tel Aviv University, Ramat Aviv 69978 Israel. E-mail: gweiss@eng.tau.ac.il 


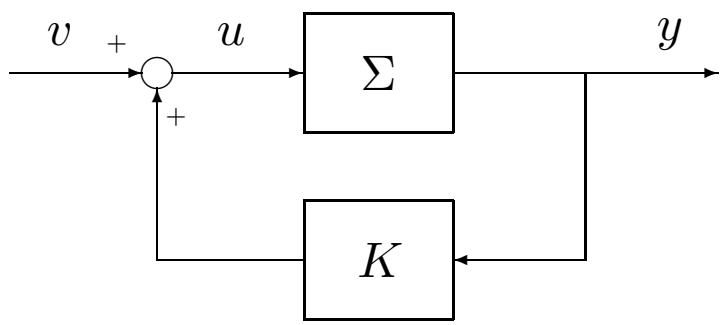

FIG. 1. A well-posed linear system $\Sigma$ with output feedback via $K$. If $K$ is admissible, then this is a new well-posed linear system $\Sigma^{K}$, called the closed-loop system.

$m \in \mathbb{N}$. A is called Riesz spectral if there is a sequence of generalized eigenvectors of $A$ which form a Riesz basis in $X$, see Dunford and Schwartz [7].

In this paper, $A$ is assumed to be diagonal. The aim of the paper is to find sufficient conditions for $A^{K}$ to be Riesz spectral. These investigations were motivated by the study of wave and beam equations with stabilizing output feedback, which have a rich literature, see for example $[2,3,4,5,24,27,31,44,45]$. If either the control operator or the observation operator of $\Sigma$ is bounded and if certain estimates are satisfied, then the Riesz basis property is preserved by output feedback, see Xu and Sallet [44]. Here we are concerned with the case where both the control operator and the observation operator are possibly unbounded. The general framework in which we look at the feedback system is as in [42]. For easy reference, the formulas needed from [42] will be written down in Sections 1 and 3.

We denote by $U$ the input space of $\Sigma$ and by $Y$ its output space; $X, U$ and $Y$ are Hilbert spaces. We denote by $B$ and $B^{K}$ the control operators of $\Sigma$ and $\Sigma^{K}$, and by $C$ and $C^{K}$ their observation operators. Thus, the state of the feedback system, denoted by $x(t)$, satisfies both of the equations

$$
\dot{x}(t)=A x(t)+B u(t), \quad \dot{x}(t)=A^{K} x(t)+B^{K} v(t),
$$

where $u=K y+v$. We have $x(t) \in X$ for all $t \geq 0, u, v \in L_{l o c}^{2}([0, \infty), U), y \in$ $L_{\text {loc }}^{2}([0, \infty), Y)$ and $K \in \mathcal{L}(Y, U)$. If we denote $X_{-1}=\mathcal{D}\left(A^{*}\right)^{\prime}$ and $X_{-1}^{K}=\mathcal{D}\left(\left(A^{K}\right)^{*}\right)^{\prime}$, both with respect to the pivot space $X$, then $B \in \mathcal{L}\left(U, X_{-1}\right)$ and $B^{K} \in \mathcal{L}\left(U, X_{-1}^{K}\right)$ (see also Section 3 for other details on $\Sigma^{K}$ ).

If $u=0$ and $x(0) \in \mathcal{D}(A)$, then $y(t)=C x(t)$ (for all $t \geq 0$ ), while if $v=0$ and $x(0) \in \mathcal{D}\left(A^{K}\right)$, then $y(t)=C^{K} x(t)$ (for all $t \geq 0$ ). If we denote $X_{1}=\mathcal{D}(A)$ and $X_{1}^{K}=\mathcal{D}\left(A^{K}\right)$, both with the graph norm, then $C \in \mathcal{L}\left(X_{1}, Y\right)$ and $C^{K} \in \mathcal{L}\left(X_{1}^{K}, Y\right)$. For general $u$ (or $v$ ) and $x(0)$, the formula for $y$ is more complicated (see [35]), but in terms of Laplace transforms we have the simple relationships

$$
\begin{aligned}
& \hat{y}(s)=C(s I-A)^{-1} x(0)+\mathbf{G}(s) \hat{u}(s), \\
& \hat{y}(s)=C^{K}\left(s I-A^{K}\right)^{-1} x(0)+\mathbf{G}^{K}(s) \hat{v}(s) .
\end{aligned}
$$


Our paper [43] is also concerned with the spectral properties of $A^{K}$, but without assuming that $A$ is diagonal. The theorem below is taken from [43] and related results were given earlier by Salamon [33] and Rebarber and Townley [28].

We need the notation $\sigma_{p}(A)$ for the point spectrum (the set of eigenvalues) of $A$. Recall that for any $\lambda \in \sigma_{p}(A), \operatorname{dim}\{\operatorname{Ker}(\lambda I-A)\}$ is called the geometric multiplicity of $\lambda$, while $\sup _{k \in \mathbb{N}} \operatorname{dim}\left\{\operatorname{Ker}(\lambda I-A)^{k}\right\}$ is called the algebraic multiplicity of $\lambda$ (see Kato [18]). The transfer function $\mathbf{G}$ is extended to $\rho(A)$, the resolvent set of $A$, as in $[35,36]$, and similarly $\mathbf{G}^{K}$ is extended to $\rho\left(A^{K}\right)$ (see also Section 3 ).

Theorem 1.1. We use the notation $\Sigma, U, X, Y, A, B, C, \mathbf{G}$ for an open-loop well-posed system, and $K, \Sigma^{K}, A^{K}, B^{K}, C^{K}, \mathbf{G}^{K}$ for an admissible feedback operator and the corresponding closed-loop system, as introduced earlier in this section (now, $A$ is not assumed to be diagonal). Assume that $\operatorname{Ker} B=\{0\}$ and $\lambda \in \rho(A)$.

Then the following statements are equivalent:

(1) $\lambda \in \sigma_{p}\left(A^{K}\right)$, i.e., $X_{\lambda}=\operatorname{Ker}\left(\lambda I-A^{K}\right) \subset \mathcal{D}\left(A^{K}\right)$ is non-trivial.

(2) $U_{\lambda}=\operatorname{Ker}[I-K \mathbf{G}(\lambda)] \subset U$ is non-trivial.

If the above statements are true, then $X_{\lambda}$ and $U_{\lambda}$ are isomorphic, via an invertible operator $T_{\lambda}: U_{\lambda} \rightarrow X_{\lambda}$. The operator $T_{\lambda}$ and its inverse $T_{\lambda}^{-1}$ are given by

$$
T_{\lambda} \mathrm{v}=(\lambda I-A)^{-1} B \mathrm{v}, \quad T_{\lambda}^{-1} x=K C^{K} x .
$$

This theorem enables us to find all the eigenvalues of $A^{K}$ which are in $\rho(A)$, by solving the "characteristic equation" (with unknowns $\lambda$ and v)

$$
[I-K \mathbf{G}(\lambda)] \mathrm{v}=0, \quad \mathrm{v} \neq 0,
$$

and then we can find the corresponding eigenvectors of $A^{K}$ using (1.2). For various related results we refer again to [43].

Systems with a diagonal semigroup have been studied extensively, and many results about the admissibilty of control (or observation) operators and various controllability (or observability) concepts have been obtained, see for example Avdonin and Ivanov [1], Hansen and Weiss [12], Ho and Russell [14], Jacob and Zwart [16], Jaffard et al [17], Rebarber and Weiss [29], Rebarber and Zwart [30] and Russell and Weiss [32]. Feedback perturbations of diagonal semigroups have been studied in Chen et al [3], Guo [9], Guo and Chan [10], Lasiecka and Triggiani [20], J.Q. Liu [21], K. Liu et al [22], Rebarber [25], Xu and Sallet [44] and many others.

Several papers deal with the problem of spectral assignability by state feedback for systems with a diagonal semigroup: see (in historical order) Sun [37], Liu [21], Ho [13], Lasiecka and Triggiani [20], Rebarber [25], Xu and Sallet [44], Sklyar and Rezounenko [34]. They all assume that the input space is one-dimensional (in [20], also finite-dimensional). They also give various sufficient conditions for the closedloop semigroup to be diagonal or Riesz spectral, and in this respect, our results 
in this paper complement their results. Recently, Guo and Luo [11] have studied systems described by a second order undamped differential equation in a Hilbert space with a scalar collocated feedback control which adds a damping term to the differential equation. They have derived sufficient conditions such that (i) the openloop system is an exactly controllable regular system and (ii) the closed-loop system generator is Riesz spectral. They have also derived asymptotic closed-loop eigenvalue and eigenvector estimates. Unfortunately, one of their assumptions implies that the open-loop spectrum gap tends to infinity, which is rather restrictive.

2. Statement of the main results. First we recall the concepts of admissible control operator and exact controllability, in the context of diagonal semigroups. Let $\mathbb{T}$ be a diagonal semigroup on the Hilbert space $X$, with generator $A$. Let $\left(\lambda_{n}\right)$ be the sequence of eigenvalues of $A$ and let $\left(e_{n}\right)$ be the corresponding sequence of eigenvectors (so that $\left(e_{n}\right)$ is a Riesz basis). Since (by the definition of a Riesz basis) there exists an invertible operator $H \in \mathcal{L}(X)$ such that $\left(H e_{n}\right)$ is an orthonormal basis in $X$, it follows that $A^{*}$ is also diagonal, with eigenvalue sequence $\left(\overline{\lambda_{n}}\right)$ and the corresponding eigenvectors

$$
\widetilde{e}_{n}=H^{*} H e_{n} .
$$

Recall that $X_{-1}$ is the dual of $\mathcal{D}\left(A^{*}\right)$ with respect to the pivot space $X$. If $A$ is diagonal and the sequence $\left(\widetilde{e}_{n}\right)$ is as in (2.1), then any $B \in \mathcal{L}\left(U, X{ }_{-1}\right)$ can be decomposed using a sequence $\left(b_{n}\right)$ in $U$, as follows:

$$
B \mathrm{v}=\sum_{n \in \mathbb{N}}\left\langle b_{n}, \mathrm{v}\right\rangle_{U} e_{n}, \quad b_{n}=B^{*} \widetilde{e}_{n}
$$

and we have

$$
\sum_{n \in \mathbb{N}} \frac{\left|\left\langle b_{n}, \mathrm{v}\right\rangle\right|^{2}}{1+\left|\lambda_{n}\right|^{2}}<\infty \quad \forall \mathrm{v} \in U .
$$

$B$ is called an admissible control operator for $\mathbb{T}$ if the $X_{-1}$-valued equation

$$
\dot{x}(t)=A x(t)+B u(t), \quad t \geq 0, \quad x(0)=0,
$$

has a continuous $X$-valued solution for any $u \in L^{2}([0, \infty), U)$, see for example Ho and Russell [14] or Hansen and Weiss [12] for details. If $B$ is admissible, then $(A, B)$ is called exactly controllable if there exists a $\tau>0$ with the following property: for any $x_{1} \in X, u$ in (2.3) can be chosen such that $x(\tau)=x_{1}$.

If $B$ is an admissible control operator for $\mathbb{T}$, then there is an $M>0$ such that

$$
\left\|b_{n}\right\|^{2} \leq M\left(1+\left|\operatorname{Re} \lambda_{n}\right|\right) \quad \forall n \in \mathbb{N} .
$$

This is [32, Proposition 4.1], stated in dual form (and without the exponential stability condition). The above condition is not sufficient for admissibility. For a detailed 
discussion of various sufficient conditions in terms of the sequence $\left(b_{n}\right)$ we refer to Hansen and Weiss [12]. If $B$ is admissible and moreover, $(A, B)$ is exactly controllable, then there is an $L>0$ such that

$$
\left\|b_{n}\right\|^{2} \geq L\left(1+\left|\operatorname{Re} \lambda_{n}\right|\right) \quad \forall n \in \mathbb{N} .
$$

This is [32, Proposition 4.2], stated in dual form (and without the exponential stability condition). Condition (2.4) is not sufficient for exact controllability. For various necessary and sufficient conditions in terms of the sequence $\left(b_{n}\right)$ (with finite-dimensional $U)$ we refer to Jacob and Zwart [16] and Tucsnak and Weiss [39].

With the notation of Theorem 1.1, assume that $A$ is diagonal and $(A, B)$ is exactly controllable. The following theorem tells us how to generate another Riesz basis for $X$ using $A$ and $B$. Our motivation is that this new Riesz basis has a good chance of being quadratically close to a sequence of linearly independent generalized eigenvectors of $A^{K}$. When this happens, then this enables us to prove the existence of a finite number of additional generalized eigenvectors of $A^{K}$ which, together with the considered sequence of generalized eigenvectors of $A^{K}$, form a Riesz basis for $X$. The details of this construction will be in Theorems 2.7 and 2.9.

Theorem 2.1. Let $A$ be the generator of a diagonal semigroup $\mathbb{T}$ on $X$, with the sequence of eigenvalues $\left(\lambda_{n}\right)$. Let $B$ be an admissible control operator for $\mathbb{T}$ such that $(A, B)$ is exactly controllable. Then, for each $r \in \mathbb{R}$ such that $\sup _{n \in \mathbb{N}} \operatorname{Re} \lambda_{n}<r / 2$, the sequence $\left(f_{n}\right)$ defined below is a Riesz basis in $X$ :

$$
f_{n}=\left[\left(r-\overline{\lambda_{n}}\right) I-A\right]^{-1} B b_{n},
$$

where $b_{n}$ is the sequence representing $B$, as in (2.2).

REMARK 2.2. There is a version of Theorem 2.1 in which we replace admissibility by infinite-time admissibility and exact controllability by exact controllability in infinite time (as defined in [12] and [32]). In this case, we may take $r=0$ in (2.5) and (after a change of sign) we obtain that the sequence $\left(f_{n}\right)$ with $f_{n}=\left(\overline{\lambda_{n}} I+A\right)^{-1} B b_{n}$ is a Riesz basis in X. The proof is similar to the proof of Theorem 2.1.

If we assume that $\mathbb{T}$ is invertible, i.e., $\mathbb{T}$ can be extended to a group acting on $X$, then we can apply Theorem 2.1 to $-A$. In particular, if $\mathbb{T}$ can be extended to a unitary group, then we obtain the following.

Corollary 2.3. Suppose that $A: \mathcal{D}(A) \rightarrow X$ has a sequence of eigenvalues $\left(i \omega_{n}\right)$ with $\omega_{n} \in \mathbb{R}$ such that a corresponding sequence of eigenvectors $\left(e_{n}\right)$ is an orthonormal basis in $X$ (thus, in particular, $A^{*}=-A$ ). Let $B$ be an admissible control operator for $\mathbb{T}$ such that $(A, B)$ is exactly controllable. Then the sequence $\left(h_{n}\right)$ given by

$$
h_{n}=\left[\left(i \omega_{n}-r\right) I-A\right]^{-1} B b_{n}
$$

(where $b_{n}$ is as in (2.2)), is a Riesz basis in $X$, for every choice of $r>0$. 
Recall that a sequence in a Hilbert space is called linearly independent if for any finite linear combination of its elements the following holds: if the coefficients are not all zero then the combination is non-zero.

TheOREM 2.4. Let $\mathcal{A}$ be a densely defined operator in a separable Hilbert space $X$, with non-empty resolvent set $\rho(\mathcal{A})$ and with compact resolvents. Let $\left(\psi_{n}\right)$ be a linearly independent sequence of generalized eigenvectors of $\mathcal{A}$ and let $\left(\widetilde{\psi}_{n}\right)$ be a Riesz basis in $X$ such that for some $m \in\{0,1,2, \ldots\}$

$$
\sum_{n \in \mathbb{N}}\left\|\psi_{n}-\widetilde{\psi}_{n+m}\right\|_{H}^{2}<\infty
$$

Then there exist $m$ generalized eigenvectors of $\mathcal{A}$, denoted by $\phi_{1}, \phi_{2}, \ldots, \phi_{m}$, such that the sequence

$$
\zeta=\left(\phi_{1}, \phi_{2}, \ldots, \phi_{m}, \psi_{1}, \psi_{2}, \psi_{3}, \ldots\right)
$$

is a Riesz basis in $X$ (in particular, $\mathcal{A}$ is Riesz spectral).

This result is essentially due to B.Z, Guo, see his Theorem 6.3 in [9]. Our statement is slightly stronger, since in our formulation $m$ in (2.8) is known. For the sake of completeness we include a proof in the next section.

REMARK 2.5. The compactness of the resolvents is essential in the last theorem. Without requiring it, the theorem is not true, even if $\mathcal{A}$ is bounded and $\left(\psi_{n}\right)$ is a sequence of eigenvectors of $\mathcal{A}$. Take for example $X=l^{2}$ with its canonical orthonormal basis $\left\{e_{n} \mid n \in \mathbb{N}\right\}$. Consider $\mathcal{A}$ defined as follows: $\mathcal{A} e_{1}=\left(1, \frac{1}{2}, \frac{1}{3}, \ldots\right)$ and $\mathcal{A} e_{n}=$ $\frac{n-1}{n} e_{n}$ for $n=2,3,4 \ldots$. Then $\sigma_{p}(\mathcal{A})=\left\{\frac{n-1}{n} \mid n=2,3,4, \ldots\right\}$. Hence, $\mathcal{A}$ has a sequence of eigenvectors $\left(\psi_{n}\right)$ with $\psi_{n}=e_{n+1} \forall n \in \mathbb{N}$, which satisfies (2.7) with $m=1$. However, $\mathcal{A}$ is not Riesz spectral.

REMARK 2.6. With the notation of Theorem 2.4, let $\left(\lambda_{n}\right)$ be the sequence of eigenvalues of $\mathcal{A}$ which corresponds to $\left(\psi_{n}\right)$ (there may be repetitions in the sequence $\left(\lambda_{n}\right)$ ). Similarly, for $j=1, \ldots, m$, let $\mu_{j}$ be the eigenvalue corresponding to $\phi_{j}$. Then $\sigma(\mathcal{A})=\sigma_{p}(\mathcal{A})=\left\{\lambda_{n} \mid n \in \mathbb{N}\right\} \cup\left\{\mu_{1}, \ldots, \mu_{m}\right\}$, as it is easy to see.

TheOREM 2.7. Let $\Sigma$ be a well-posed linear system with semigroup generator $A$ and control operator $B$, which satisfy the assumptions of Corollary 2.3 (in particular, $\mathbb{T}$ is unitary and $(A, B)$ is exactly controllable). The sequence $\left(h_{n}\right)$ is defined as in (2.6). Assume that $K$ is an admissible feedback operator for $\Sigma$ such that $A^{K}$ has compact resolvents. Let $\Lambda=\left\{\sigma_{n} \in \mathbb{C} \mid n \in \mathbb{N}\right\}$ denote a subset of $\sigma\left(A^{K}\right)$ contained in $\rho(A)$. Let $\left(\psi_{n}\right)$ be a linearly independent sequence of generalized eigenvectors of $A^{K}$ corresponding to $\Lambda$. If there are some integer $m \in\{0,1,2, \ldots\}$ and some $r>0$ in (2.6) such that

$$
\sum_{k \in \mathbb{N}}\left\|\psi_{k}-h_{k+m}\right\|_{X}^{2}<\infty
$$


then there exist $m$ generalized eigenvectors of $A^{K}, \phi_{1}, \phi_{2}, \ldots, \phi_{m}$ which, concatenated with $\left(\psi_{n}\right)$ like in (2.8), form a Riesz basis in $X$.

REMARK 2.8. From the identity (3.4) in the next section, if $U$ or $Y$ is finitedimensional, then $A^{K}$ has compact resolvents if and only if $A$ does. From (3.2)-(3.4), if $B$ or $C$ is bounded, then again $A$ has compact resolvents if and only if $A^{K}$ does.

With the notation of the last theorem, consider the case $U=\mathbb{C}$. The rank of the observation operator $C$ is not important here. In this case, $b_{n}$ in (2.6) is a non-zero number. We may take $\mathrm{v}=b_{n}$ in (1.2), obtaining the following sequence of eigenvectors of $A^{K}$ :

$$
\psi_{n}=\left(\sigma_{n} I-A\right)^{-1} B b_{n} .
$$

In this case we get a practical sufficient condition from the last theorem:

TheOREM 2.9. With the assumptions and the notation of Theorem 2.7, suppose that $U=\mathbb{C}$. Let $\Lambda=\left\{\sigma_{n} \in \mathbb{C} \mid n \in \mathbb{N}\right\}$ be a subset of $\sigma\left(A^{K}\right)$ contained in $\rho(A)$ such that $\sigma_{n} \neq \sigma_{k}, \forall n \neq k$. If, for some $m \in\{0,1,2, \ldots\}$ and some $r>0$, the following holds:

$$
\sum_{n \in \mathbb{N}}\left|-r+i \omega_{n+m}-\sigma_{n}\right|^{2}<\infty
$$

then $A^{K}$ is Riesz spectral. Moreover, in this case there exist $m$ eigenvalues of $A^{K}$, $\widetilde{\sigma}_{1}, \ldots, \widetilde{\sigma}_{m}$, such that

$$
\sigma\left(A^{K}\right)=\Lambda \bigcup\left\{\widetilde{\sigma}_{1}, \ldots, \widetilde{\sigma}_{m}\right\}
$$

Remark 2.10. If $A, B$ and $\omega_{n}$ are as in Corollary 2.3 and $U=\mathbb{C}$, then the numbers $\omega_{n}$ must have a uniform gap (this is easy to check, see also [16]). This implies that $A$ has compact resolvents. By Remark 2.8, the same is true for $A^{K}$, so that there is no need to verify this condition in Theorem 2.7 .

In some applications the set $\sigma\left(A^{K}\right)$ may asymptotically approach $\sigma(A)$ shifted to the left by $r$ (for example, in the stabilization of vibrating systems by collocated output feedback, see Sections 4 and 5). If the control input is scalar (i.e., $U=\mathbb{C}$ ), then Theorem 2.9 can be useful. However, for some feedback stabilized hyperbolic systems, such as in Rebarber [27], the spectrum of $A^{K}$ may not even approach any vertical line. Nevertheless $\sigma\left(A^{K}\right)$ asymptotically remains in a vertical strip: for large $n, \delta_{1} \leq \operatorname{Re}\left(\sigma_{n}\right) \leq \delta_{2}$ with $\delta_{1}<\delta_{2}$. The next theorem has been developed in order to prove Riesz spectralness for such feedback systems.

Theorem 2.11. Let $\Sigma$ be well-posed with input space $U=\mathbb{C}$, state space $X$, semigroup generator $A$ and control operator $B$. Suppose that $A$ is diagonal, $\sigma(A)=$ $\left\{ \pm i \omega_{n} \mid \omega_{n} \in(0, \infty), n \in \mathbb{N}\right\}$ (in increasing order: $\omega_{n+1}>\omega_{n}$ ) satisfying

$$
\sum_{n \in \mathbb{N}} \frac{1}{\left(\omega_{n+1}-\omega_{n}\right)^{2}}<\infty
$$


$(A, B)$ is exactly controllable and $K$ is an admissible feedback operator for $\Sigma$. Let $\Sigma^{K}$ be the corresponding closed-loop system, with semigroup generator $A^{K}$. Let $\left(\sigma_{n}\right)$ be a sequence in $\sigma\left(A^{K}\right) \cap \rho(A)$ such that $\operatorname{Im} \sigma_{n}>0, \sigma_{n} \neq \sigma_{k} \quad \forall n \neq k$ and $\overline{\sigma_{n}} \in$ $\sigma\left(A^{K}\right) \cap \rho(A)$. If, for some integer $m \geq 0$,

$$
\sum_{n \in \mathbb{N}}\left(\operatorname{Im} \sigma_{n}-\omega_{n+m}\right)^{2}<\infty
$$

then $A^{K}$ is Riesz spectral and there are $2 m$ complex numbers $\widetilde{\sigma}_{1}, \ldots, \widetilde{\sigma}_{2 m}$ such that $\sigma\left(A^{K}\right)=\left\{\widetilde{\sigma}_{1}, \ldots, \widetilde{\sigma}_{2 m}\right\} \cup\left\{\sigma_{n} \mid n \in \mathbb{N}\right\} \cup\left\{\overline{\sigma_{n}} \mid n \in \mathbb{N}\right\}$.

We will illustrate the usefulness of these results through several examples concerning the stabilization of systems described by partial differential equations (PDEs) in one dimension. For these systems we show that the closed-loop generator is Riesz spectral. Our approach leads to some generalizations of existing results and a unified treatment of different cases which have been studied separately, see the references already mentioned. It also enables us to simplify certain computations. The exact controllability (or the exact observability) condition which needs to be verified in our approach can be tested by powerful methods such as the multiplier method, see for example Komornik [19], or the recent criteria of Jacob and Zwart [16]. Our method is very efficient in dealing with systems governed by PDEs coupled with ordinary differential equations, leading to positive answers to several open questions in the literature, see the examples in Sections 4 and 5.

3. Proof of the main results. Throughout this section, $X$ is a Hilbert space and $A: \mathcal{D}(A) \rightarrow X$ is the generator of a strongly continuous semigroup $\mathbb{T}$ on $X$. The Hilbert space $X_{1}$ is $\mathcal{D}(A)$ with the norm $\|z\|_{1}=\|(\beta I-A) z\|$, where $\beta \in \rho(A)$ is fixed (this norm is equivalent to the graph norm). The Hilbert space $X_{-1}$ is the completion of $X$ with respect to the norm $\|z\|_{-1}=\left\|(\beta I-A)^{-1} z\right\|$. This space is isomorphic to $\mathcal{D}\left(A^{*}\right)^{\prime}$, the dual space of $\mathcal{D}\left(A^{*}\right)$ and

$$
X_{1} \subset X \subset X_{-1},
$$

densely and with continuous embeddings. $\mathbb{T}$ extends to a semigroup on $X_{-1}$, denoted by the same symbol. The generator of this extended semigroup is an extension of $A$, whose domain is $X$, so that $A: X \rightarrow X_{-1}$. We consider a well-posed linear system $\Sigma$ with input space $U$, state space $X$, output space $Y$, semigroup generator $A$, control operator $B$, observation operator $C$ and transfer function $\mathbf{G}$. Thus, $B \in \mathcal{L}\left(U, X_{-1}\right)$ is an admissible control operator for $\mathbb{T}$ and $C \in \mathcal{L}\left(X_{1}, Y\right)$ is an admissible observation operator for $\mathbb{T}$. For the various concepts mentioned above we refer to [36, 40, 41, 42] and the references therein. $B$ is called bounded if $B \in \mathcal{L}(U, X)$, and $C$ is called bounded if it can be extended such that $C \in \mathcal{L}(X, Y)$.

Denote the growth bound of $\mathbb{T}$ by $\omega_{0}(\mathbb{T})$. Originally, $\mathbf{G}$ is defined on the right 
half-plane where $\operatorname{Re} s>\omega_{0}(\mathbb{T})$. For all $s, \beta$ in this half-plane, we have

$$
\mathbf{G}(s)-\mathbf{G}(\beta)=C\left[(s I-A)^{-1}-(\beta I-A)^{-1}\right] B .
$$

Using the above formula with $\operatorname{Re} \beta>\omega_{0}(\mathbb{T})$ but allowing $s \in \rho(A)$, we extend $\mathbf{G}$ to an analytic function defined on $\rho(A)$. Then, this function $\mathbf{G}$ satisfies (3.1) for all $s, \beta \in \rho(A)$. This extension of $\mathbf{G}$ has been used in $[33,35,36]$ and it may be different from the extension by analytic continuation, adopted in [41, 42] (see [41, Remark 4.8]). However, if $\rho(A)$ is connected, then this extension of $\mathbf{G}$ is the same as its analytic continuation. The function $\mathbf{G}$ is well-posed, which means that it is a bounded and analytic $\mathcal{L}(U, Y)$-valued function on some right half-plane (in particular, on any half-plane which is strictly to the right of $\left.\omega_{0}(\mathbb{T})\right)$.

An operator $K \in \mathcal{L}(Y, U)$ is called an admissible feedback operator for $\Sigma$ (or for $\mathbf{G})$ if $I-\mathbf{G} K$ is invertible on some right half-plane and its inverse is well-posed (here, $I-\mathbf{G} K$ may be replaced equivalently by $I-K \mathbf{G}$ ). If this is the case, then the feedback system from Figure 1 is a new well-posed linear system $\Sigma^{K}$. We use the notation $A^{K}, B^{K}, C^{K}$ and $\mathbf{G}^{K}$ as introduced in Section 1, so that in particular (1.1) holds on some right half-plane. What is less obvious is that (1.1) remains valid for all $s \in \rho(A) \cap \rho\left(A^{K}\right)$ (in particular, $I-K \mathbf{G}(s)$ is invertible for all such $s$ ). Indeed, this follows from Corollary 5.3 and Remark 5.5 in [36]. Let $\mathbb{T}^{K}$ denote the strongly continuous semigroup generated by $A^{K}$. The following identities, proved in [42], will be useful:

$$
\begin{aligned}
& {[I-\mathbf{G}(s) K] C^{K}\left(s I-A^{K}\right)^{-1}=C(s I-A)^{-1},} \\
& \left(s I-A^{K}\right)^{-1} B^{K}[I-K \mathbf{G}(s)]=(s I-A)^{-1} B \\
& \left(s I-A^{K}\right)^{-1}-(s I-A)^{-1}=(s I-A)^{-1} B K C^{K}\left(s I-A^{K}\right)^{-1} \\
& =\left(s I-A^{K}\right)^{-1} B^{K} K C(s I-A)^{-1} .
\end{aligned}
$$

According to [42], these identities are valid on the right half-plane where $\operatorname{Re} s>$ $\max \left\{\omega_{0}(\mathbb{T}), \omega_{0}\left(\mathbb{T}^{K}\right)\right\}$. We remark that, using again [36, Section 5], it follows that (3.2)-(3.4) remain valid for all $s \in \rho(A) \cap \rho\left(A^{K}\right)$. Note that (3.3) implies that $\operatorname{Ker} B=$ $\{0\}$ if and only if $\operatorname{Ker} B^{K}=\{0\}$. It is worth noting that (3.4) implies (via an easy computation) that for all $x \in \mathcal{D}\left(A^{K}\right)$ and for all $z \in \mathcal{D}(A)$,

$$
A^{K} x=\left(A+B K C^{K}\right) x, \quad A z=\left(A^{K}-B^{K} K C\right) z .
$$

It is not difficult to prove, using (3.2), that $C^{K}$ is bounded if and only if $C$ is bounded. Similarly, (3.3) implies that $B^{K}$ is bounded if and only if $B$ is bounded. 
An important subclass of the well-posed linear systems are the regular linear systems, see for example [41, 42]. The system $\Sigma$ is called regular if for each $\mathrm{v} \in U$, the limit

$$
D \mathrm{v}=\lim _{\lambda \rightarrow+\infty} \mathbf{G}(\lambda) \mathrm{v}
$$

exists (in $Y$ ). The operator $D \in \mathcal{L}(U, Y)$ is then called the feedthrough operator of $\Sigma$. We define the $\Lambda$-extension of $C$ by

$$
C_{\Lambda} x=\lim _{\lambda \rightarrow+\infty} C \lambda(\lambda I-A)^{-1} x \quad \forall x \in \mathcal{D}\left(C_{\Lambda}\right),
$$

where $\mathcal{D}\left(C_{\Lambda}\right)$ is the space of those $x \in X$ for which the above limit exists. The system $\Sigma$ is regular if and only if $(s I-A)^{-1} B U \subset \mathcal{D}\left(C_{\Lambda}\right)$ for some (hence, for every) $s \in \rho(A)$ and, if this is the case, then $\mathbf{G}(s)=C_{\Lambda}(s I-A)^{-1} B+D$ for all $s \in \rho(A)$. Moreover, for a regular system, $y(t)=C_{\Lambda} x(t)+D u(t)$ holds for almost every $t \geq 0$ (for every initial state $x(0) \in X$ and every input signal $\left.u \in L_{l o c}^{2}([0, \infty), U)\right)$.

If $\Sigma$ is regular, $K$ is an admissible feedthrough operator for $\Sigma$ and $I-D K$ is invertible, then $\Sigma^{K}$ is also regular and the relationship between the open and closedloop systems becomes more transparent. Indeed, in this case $A^{K}, B^{K}, C^{K}$ and $D^{K}$ can be expressed in terms of $A, B, C$ and $D$, see [42], in particular

$$
A^{K} x=\left[A+B K(I-D K)^{-1} C_{\Lambda}\right] x, \quad C^{K} x=(I-D K)^{-1} C_{\Lambda} x,
$$

for all $x \in \mathcal{D}\left(A^{K}\right)$. Thus, regularity simplifies matters, and it will be useful in the examples, however, in general we will only assume the well-posedness of $\Sigma$.

The following simple facts from Russell and Weiss [32] will be needed.

Proposition 3.1. Let $X$ and $Y$ be Hilbert spaces. Assume that $A$ is the generator of a $C_{0}$-semigroup $\mathbb{T}$ on $X$ and $C: \mathcal{D}(A) \rightarrow Y$ is an admissible observation operator for $\mathbb{T}$. Then the following statements hold:

1. For any $\lambda \in \mathbb{C}$, the pair $(A, C)$ is exactly observable if and only if the translated pair $(A+\lambda I, C)$ is exactly observable.

2. If $\mathbb{T}$ is exponentially stable, then $(A, C)$ is exactly observable if and only if it is exactly observable in infinite time.

3. If $\mathbb{T}$ is exponentially stable, then the observability Gramian of $(A, C)$, defined by

$$
P x=\int_{0}^{\infty} \mathbb{T}_{t}^{*} C^{*} C \mathbb{T}_{t} x \mathrm{~d} t \quad \forall x \in \mathcal{D}(A)
$$

can be extended so that $P \in \mathcal{L}(X) . P$ is invertible if and only if $(A, C)$ is exactly observable.

4. If $\mathbb{T}$ is a $C_{0}$-group on $X$ and $(A, C)$ is exactly observable, there exist some positive constants $\alpha$ and $\beta$ such that the following holds: For any normalized eigenvector $e_{k}$ of $A$,

$$
\alpha \leq\left\|C e_{k}\right\|_{Y} \leq \beta
$$


Proof of Theorem 2.1. We introduce the "shifted" generator $\tilde{A}=A-\frac{r}{2} I$, which generates an exponentially stable semigroup. For simplicity we denote this semigroup by $e^{\tilde{A} t}$ and similarly for $\tilde{A}^{*}$. The pair $(A, B)$ is exactly controllable if and only if $\left(A^{*}, B^{*}\right)$ is exactly observable. From our assumptions and point 1 of Proposition 3.1, $\left(\tilde{A}^{*}, B^{*}\right)$ is exactly observable. Hence, $\left(\tilde{A}^{*}, B^{*}\right)$ is exactly observable in infinite time. The observability Gramian $P$ of $\left(\tilde{A}^{*}, B^{*}\right)$ is invertible (point 3 of Proposition 3.1). Hence, applying $P$ to each $\widetilde{e}_{n}$ we obtain a new basis:

$$
P \widetilde{e}_{n}=\int_{0}^{\infty} e^{\tilde{A} t} B B^{*} e^{\tilde{A}^{*} t} \widetilde{e}_{n} \mathrm{~d} t=\int_{0}^{\infty} e^{-t\left(r-\overline{\lambda_{n}}\right)} \mathbb{T}_{t} B B^{*} \widetilde{e}_{n} \mathrm{~d} t .
$$

Using the Laplace transform of $\mathbb{T}_{t}$ we get $P \widetilde{e}_{n}=f_{n}$, for all $n \in \mathbb{N}$.

Remark 3.2. Without assuming that $\mathbb{T}$ is exponentially stable, suppose that $C$ is infinite-time admissible for $\mathbb{T}$, so that $t \rightarrow C_{\Lambda} \mathbb{T}_{t} x$ is a function in $L^{2}([0, \infty), Y)$. By duality, the infinite-time admissibility of $C$ implies two facts: (a) For each $T>0$, the mapping $\Phi_{T}: u \rightarrow \int_{0}^{T} \mathbb{T}_{t}^{*} C^{*} u(t) \mathrm{d} t$ is continuous from $L^{2}([0, \infty), Y)$ to $X$ and (b) The operators $\Phi_{T}$ converge strongly as $T \rightarrow+\infty$. Thus, the integral in (3.9) should now be considered as an improper integral in $X$, and $P$ is invertible if and only if $(A, C)$ is exactly observable in infinite time. As a consequence, if $(A, C)$ is exactly observable in infinite time (3.2) gives us a Riesz basis with $r=0$, see Remark 2.2.

Lemma 3.3. Let $R$ be a densely defined operator in a separable Hilbert space $X$ with compact resolvents and let $\mathcal{C}$ be the closed linear span of all the generalized eigenvectors of $R$. If $\mathcal{C}$ is of finite codimension in $X$, then $\mathcal{C}=X$.

Proof. An operator $R$ as in the lemma is called a discrete operator in Dunford and Schwartz [7], and $\mathcal{C}$ is called the spectral span of $R$. It is easy to see that $\sigma(R)=\sigma_{p}(R)$ and $\sigma\left(R^{*}\right)=\sigma_{p}\left(R^{*}\right)$. According to [7, Lemma 5 on p. 2355], the following orthogonal decomposition holds:

$$
X=\sigma_{\infty}\left(R^{*}\right) \oplus \mathcal{C}
$$

where $\sigma_{\infty}\left(R^{*}\right)=\left\{x \mid P_{\lambda} x=0 \forall \lambda \in \sigma\left(R^{*}\right)\right\}$ and $P_{\lambda}$ is the eigen-projector of $R^{*}$ corresponding to $\lambda$. Hence, $\mathcal{C}=X$ if and only if $\sigma_{\infty}\left(R^{*}\right)=\{0\}$. However, by [7, Lemma 5 on p. 2295], $\sigma_{\infty}\left(R^{*}\right)$ is either $\{0\}$ or infinite-dimensional. Therefore, the codimension of $\mathcal{C}$ is finite if and only if $\sigma_{\infty}\left(R^{*}\right)=\{0\}$.

A sequence $\left(e_{n}\right)$ in a Hilbert space $X$ is called $\omega$-independent if the equality $\sum_{k \in \mathbb{N}} a_{k} e_{k}=0$ with $a_{k} \in \mathbb{C}$ implies that $a_{k}=0$ for all $k$ (see [8, p. 317]). Here, the series $\sum_{k \in \mathbb{N}} a_{k} e_{k}$ is supposed to converge in norm, but not necessarily absolutely. Two sequences $\left(\phi_{n}\right)$ and $\left(\psi_{n}\right)$ in $X$ are called quadratically close if $\sum_{n \in \mathbb{N}}\left\|\phi_{n}-\psi_{n}\right\|_{X}^{2}<$ $+\infty$. A sequence $\left(e_{n}\right)$ in $X$ called a Riesz basis in $X$ if for each $x \in X$ there exists a unique sequence $\left(a_{k}\right) \in l^{2}$ such that $x=\sum_{k \in \mathbb{N}} a_{k} e_{k}$ and

$$
c_{1}\left\|\left(a_{k}\right)\right\|_{l^{2}} \leq\|x\| \leq c_{2}\left\|\left(a_{k}\right)\right\|_{l^{2}},
$$


where $c_{1}, c_{2}>0$ are independent of $x$. For another characterization of Riesz bases see the text before (2.1). We recall Bari's theorem: an $\omega$-independent sequence quadratically close to a Riesz basis is itself a Riesz basis (see [8, p.317]).

Proof of Theorem 2.4. Let $m$ be as in (2.7). For some integer $J \geq 0$ which will be chosen later, we define the sequence $\xi=\left(\xi_{n}\right)$ in $X$ by

$$
\xi=\left(\widetilde{\psi}_{1}, \widetilde{\psi}_{2}, \ldots \widetilde{\psi}_{m+J}, \psi_{J+1}, \psi_{J+2}, \ldots\right) \text {. }
$$

Note that for $n>m+J$ we have $\xi_{n}=\psi_{n-m}$, which is close to $\widetilde{\psi}_{n}$ by (2.7).

We claim that $\left(\xi_{n}\right)$ is a Riesz basis in $X$ for sufficiently large $J$. Indeed, define $T: X \rightarrow X$ such that $T \widetilde{\psi}_{n}=\xi_{n} \forall n \in \mathbb{N}$. It suffices to prove that $T$ is continuous and invertible. We decompose $T=I+\Theta$, where

$$
\Theta \widetilde{\psi}_{n}= \begin{cases}0 & \text { for } 1 \leq n \leq J+m \\ \psi_{n-m}-\widetilde{\psi}_{n} & \text { for } n>J+m .\end{cases}
$$

Since $\|\Theta\| \leq \widetilde{K}\left(\sum_{k \in \mathbb{N}}\left\|\Theta \widetilde{\psi}_{k}\right\|^{2}\right)^{\frac{1}{2}}$ for some constant $\widetilde{K}>0$, by the quadratic closeness condition (2.7) we have $\Theta \in \mathcal{L}(X)$ and $\|\Theta\|_{\mathcal{L}(X)}<1$ for all sufficiently large $J$. This means that for some $J, T$ is boundedly invertible. Therefore, by the definition of $T,\left(\xi_{n}\right)$ is a Riesz basis in $X$.

In the sequel, we assume that $J$ has been chosen such that $\xi$ is a Riesz basis. Then it follows that the closed linear space $\mathcal{C}_{J}$ spanned by $\left(\psi_{n}\right)_{n>J}$ has finite codimension in $X$, equal to $J+m$. In particular, the closed linear space $\mathcal{C}$ spanned by all the generalized eigenvectors of $\mathcal{A}$ has finite codimension in $X$, so that $\mathcal{C}=X$ by Lemma 3.3. Thus, the closed linear span of all the generalized eigenvectors of $\mathcal{A}$ is $X$.

Since the codimension of the closed linear span $\mathcal{C}_{J}$ of the linearly independent subsequence $\left(\psi_{n}\right)_{n>J}$ is equal to $J+m$, there must exist $J+m$ generalized eigenvectors of $\mathcal{A}$ which, concatenated with the subsequence $\left(\psi_{n}\right)_{n>J}$, form a linearly independent sequence. In fact, for the first $J$ generalized eigenvectors of $\mathcal{A}$ we may take $\psi_{k}$, $k=1,2, \ldots, J$. The existence of the other $m$ generalized eigenvectors, denoted by $\phi_{k}, k=1,2, \ldots, m$, is guaranteed by $\mathcal{C}=X$, which was proved earlier. Note that by construction the sequence $\zeta$ from (2.8) is linearly independent. Since $\mathcal{A}$ has compact resolvents, the sequence $\zeta$ is necessarily $\omega$-independent (see Xu and Sallet [44, Section $3]$ ). Obviously, the sequence $\zeta$ is quadratically close to $\xi$. From Bari's theorem $\zeta$ is also a Riesz basis in $X$.

Proof of Theorem 2.9. Denote $b_{k}=B^{*} e_{k}$. From point 4 of Proposition 3.1 and the exact controllability of $(A, B)$ there exist $\alpha, \beta>0$ such that $\alpha \leq\left|b_{k}\right| \leq \beta \quad \forall k \in \mathbb{N}$. We have $\Lambda \subset \rho(A) \cap \sigma_{p}\left(A^{K}\right)$. By (2.10) and $\sigma_{n} \neq \sigma_{k}$ for $n \neq k$ the sequence $\left(\psi_{n}\right)$ defined below is a linearly independent sequence of eigenvectors of $A^{K}$ :

$$
\psi_{n}=\left(\sigma_{n} I-A\right)^{-1} B b_{n+m} .
$$


By Corollary 2.3 the sequence $\left(h_{n}\right)$ given in $(2.6)$ is a Riesz basis in $X$. Recall the following identity:

$$
\psi_{n}-h_{n+m}=\left(i \omega_{n+m}-r-\sigma_{n}\right)\left(\sigma_{n} I-A\right)^{-1}\left[\left(i \omega_{n+m}-r\right) I-A\right]^{-1} B b_{n+m} .
$$

Because of (2.11) we have $\lim _{n \rightarrow \infty} \operatorname{Re}\left(\sigma_{n}\right)=-r$. By $r>0$ and admissibility of $B$,

$$
\sup _{n \in \mathbb{N}}\left\|\left(\sigma_{n} I-A\right)^{-1}\right\|_{\mathcal{L}(X)}<\infty, \quad \sup _{n \in \mathbb{N}}\left\|\left[\left(i \omega_{n}-r\right) I-A\right]^{-1} B\right\|_{X}<\infty .
$$

Thus, the condition (2.11) and (3.11) imply that (2.9) holds. According to Theorem 2.7 and Remarks 2.6 and 2.8, the conclusions of Theorem 2.9 hold.

A sequence $\left(\lambda_{n}\right)$ in $\mathbb{C}$ is called properly spaced if

$$
\sup \operatorname{Re} \lambda_{n}=\alpha<\infty \quad \text { and } \quad \inf _{\substack{k, n \in \mathbb{N} \\ k \neq n}} \frac{\left|\lambda_{k}-\lambda_{n}\right|}{1+\left|\operatorname{Re} \lambda_{n}\right|}>0 .
$$

Note that if $\alpha<0$, then the term 1 in the denominator above can be omitted without affecting the meaning of the condition: $\inf _{\substack{k, n \in \mathbb{N} \\ k \neq n}} \frac{\left|\lambda_{k}-\lambda_{n}\right|}{\left|\operatorname{Re} \lambda_{n}\right|}>0$. This is how the concept was defined in [32, Section 4], while investigating exact observability for systems with an exponentially stable diagonal semigroup. If we write the condition like this but allow $\alpha=0$, as in Jacob and Zwart [16], then the meaning changes (the points could be very close to each other near the imaginary axis).

Proposition 3.4. Let $A$ be the generator of a diagonal semigroup $\mathbb{T}$ on $X$, with the sequence of its eigenvalues $\left(\lambda_{n}\right)$ properly spaced. Assume that $U$ is finite-dimensional and $B \in \mathcal{L}\left(U, X_{-1}\right)$ is an admissible control operator for $\mathbb{T}$. Let $\left(b_{n}\right)$ be the sequence in $U$ representing $B$, as in $(2.2)$. Then $(A, B)$ is exactly controllable if and only if there exists an $L>0$ such that (2.4) holds.

Proof. Without loss of generality, we may assume that $\mathbb{T}$ is exponentially stable. Indeed, if it not, then replace $A$ by $A-\omega I$ for some sufficiently large $\omega>0$. The admissibility of $B$ for $\mathbb{T}$ is equivalent to the admissibility of $B$ for the semigroup generated by $A-\omega I$, and the exact controllability of $(A, B)$ is equivalent to the exact controllability of $(A-\omega I, B)$. Now, with the exponential stability, (2.4) is equivalent to:

$$
\left\|b_{n}\right\|^{2} \geq L\left|\operatorname{Re} \lambda_{n}\right| \quad \forall n \in \mathbb{N} .
$$

By [32, Theorem 4.4] (which assumes that $\left(\lambda_{n}\right)$ is properly spaced), the above estimate is equivalent to

$$
\frac{1}{|\operatorname{Re} s|^{2}}\left\|\left(s I-A^{*}\right) x\right\|^{2}+\frac{1}{|\operatorname{Re} s|}\left\|B^{*} x\right\|^{2} \geq q\|x\|^{2}
$$

for some $q>0$, for all $x \in \mathcal{D}\left(A^{*}\right)$ and all $s$ in the open left half-plane in $\mathbb{C}$. Now by Theorem 2 in Jacob and Zwart [16] (which assumes that $A$ is diagonal and $U$ is 
finite-dimensional), the last estimate is equivalent to

$$
\int_{0}^{\infty}\left\|B^{*} \mathbb{T}_{t}^{*} x\right\|^{2} \mathrm{~d} t \geq p\|x\|^{2}
$$

for some $p>0$, for all $x \in \mathcal{D}\left(A^{*}\right)$. By part 2 of Proposition 3.1, (3.12) is equivalent to $\left(A^{*}, B^{*}\right)$ being exactly observable, i.e., $(A, B)$ is exactly controllable.

As already mentioned, (2.4) is a necessary condition for exact controllability also if $\left(\lambda_{n}\right)$ is not properly spaced, and for any Hilbert space $U$. We suspect that Proposition 3.4 remains valid also for an infinite-dimensional Hilbert space $U$.

Remark 3.5. A sequence $\left(\lambda_{n}\right)$ in the open left half-plane satisfies the Carleson condition if

$$
\prod_{j \in \mathbb{N}, j \neq k}\left|\frac{\lambda_{k}-\lambda_{j}}{\lambda_{k}+\overline{\lambda_{j}}}\right| \geq \delta>0, \quad \forall k \in \mathbb{N} .
$$

For sequences $\left(\lambda_{n}\right)$ with $\operatorname{Re} \lambda_{n}<0$, this condition is a little more restrictive than being properly spaced. Under the assumptions of Proposition 3.4, if $\mathbb{T}$ is exponentially stable and $(A, B)$ is exactly controllable, then $\left(\lambda_{n}\right)$ satisfies the Carleson condition. The outline of the proof of this fact is as follows. By [12, Theorem 1.2], $B$ satisfies the operator Carleson measure criterion for $\mathbb{T}$, as defined there. Let $\mathrm{v}_{1}, \ldots, \mathrm{v}_{p}$ be an orthonormal basis in $U$ (we have denoted $p=\operatorname{dim} U$ ). It is not difficult to prove that for any finite set $J \subset \mathbb{N}$

$$
\sup _{\|\mathrm{v}\| \leq 1} \sum_{k \in J}\left|\left\langle b_{k}, \mathrm{v}\right\rangle\right|^{2} \geq \frac{1}{p} \sum_{k \in J}\left\|b_{k}\right\|^{2}
$$

and this implies that the sequence $\left(\left\|b_{n}\right\|\right)$ satisfies the Carleson measure criterion for $\mathbb{T}$. By the scalar Carleson measure criterion (see Ho and Russell [14]), this sequence defines an admissible control operator for $\mathbb{T}$, for scalar inputs. By (2.4), the same is true for the sequence $\left(b_{k}^{1}\right)$ defined by $b_{k}^{1}=\sqrt{\left|\operatorname{Re} \lambda_{k}\right|}$. Now the claim made earlier in this remark follows from results in Nikolskii [23], as explained in [29, Remark 2.3].

Theorem 3.6. Let $A$ be the generator of a diagonal semigroup $\mathbb{T}$ on $X$, with the sequence of eigenvalues $\left(\lambda_{n}\right)$. Let $\left(\mu_{n}\right)$ be a properly spaced sequence in $\mathbb{C}$ such that

$$
\sup _{n \in \mathbb{N}}\left|\operatorname{Re}\left(\mu_{n}-\lambda_{n}\right)\right|<\infty
$$

Let $A^{\mu}$ be the generator of a diagonal semigroup $\mathbb{T}^{\mu}$ on $X$, so that the sequence of eigenvalues of $A^{\mu}$ is $\left(\mu_{n}\right)$ and the corresponding eigenvectors are the same as for A. Assume that $U$ is finite-dimensional and $B \in \mathcal{L}\left(U, X_{-1}\right)$ is an admissible control operator for both $\mathbb{T}$ and $\mathbb{T}^{\mu}$, such that $(A, B)$ is exactly controllable. Then also $\left(A^{\mu}, B\right)$ is exactly controllable.

Proof. Since $B$ is admissible for $\mathbb{T}$ and $(A, B)$ is exactly controllable, by [32, Proposition 4.2] (applied to $\left(A^{*}-\omega I, B^{*}\right)$ with $\omega>0$ sufficiently large) we have 
(2.4). The fact that $\sup \left|\operatorname{Re}\left(\mu_{n}-\lambda_{n}\right)\right|<\infty$ implies that for some $L_{0}>0$,

$$
\left\|b_{n}\right\|^{2} \geq L_{0}\left(1+\left|\operatorname{Re} \mu_{n}\right|\right) \quad \forall n \in \mathbb{N} .
$$

Now by Proposition $3.4,\left(A^{\mu}, B\right)$ is exactly controllable.

REMARK 3.7. With the assumptions and the notation of the above theorem, it follows from Theorem 2.1 that the sequence

$$
\widetilde{f}_{n}=\left[\left(r-\bar{\mu}_{n}\right) I-A^{\mu}\right]^{-1} B b_{n}
$$

is a Riesz basis in $X$. Here, $r \in \mathbb{R}$ is such that $\sup _{n \in \mathbb{N}} \operatorname{Re} \mu_{n}<r / 2$ and $\left(\widetilde{e}_{n}\right)$ is the sequence of eigenvectors of $A^{*}$ in the correct order, as in (2.1). If it is possible to take $r=0$ (for exemple, if $A^{\mu}$ is exponentially stable), then we may consider the Riesz basis defined by

$$
f_{n}=\left(\bar{\mu}_{n} I+A^{\mu}\right)^{-1} B b_{n}
$$

It is an unpleasant feature of Theorem 3.6 that we have to check the admissibility of $B$ also for $\mathbb{T}^{\mu}$. This inconvenience disappears if the eigenvalues are in a vertical strip. Note that a sequence $\left(\mu_{n}\right)$ in a vertical strip (i.e., $\gamma \leq \operatorname{Re} \mu_{n} \leq \alpha$ ) is properly spaced if and only if $\inf _{k \neq n}\left|\mu_{k}-\mu_{n}\right|>0$. The following theorem is a version of Theorem 3.6 for eigenvalues in a vertical strip.

Theorem 3.8. Let $A$ be the generator of a diagonal semigroup $\mathbb{T}$ on $X$, with the sequence of eigenvalues $\left(\lambda_{n}\right)$ in a vertical strip. Let $\left(\mu_{n}\right)$ be a properly spaced sequence in a vertical strip. Let $A^{\mu}$ be the generator of a diagonal semigroup $\mathbb{T}^{\mu}$ on $X$, so that the sequence of eigenvalues of $A^{\mu}$ is $\left(\mu_{n}\right)$ and the corresponding eigenvectors are the same as for $A$ (in the same order).

Assume that $U$ is finite-dimensional and $B \in \mathcal{L}\left(U, X_{-1}\right)$ is an admissible control operator for $\mathbb{T}$ such that $(A, B)$ is exactly controllable. Then the following holds:

(1) $B$ is admissible for $\mathbb{T}^{\mu}$,

(2) $\left(A^{\mu}, B\right)$ is exactly controllable.

Proof. Since $B$ is admissible for $\mathbb{T}$, it follows from [32, Proposition 4.1] (applied to $\left(A^{*}-\omega I, B^{*}\right)$ with $\omega>0$ sufficiently large) that we have $\left\|b_{n}\right\|^{2} \leq M$ for some $M>0$ and all $n \in \mathbb{N}$. Since $\left(\mu_{n}\right)$ is properly spaced, it follows from [12, Theorem 2.6] that $B$ is an admissible control operator for $\mathbb{T}^{\mu}$. The exact controllability of $\left(A^{\mu}, B\right)$ follows from Theorem 3.6.

REMARK 3.9. For $A$ diagonal and skew-adjoint such that $A e_{n}=i \omega_{n} e_{n}$, we consider the diagonal $A^{\mu}$ such that $A^{\mu} e_{n}=\mu_{n} e_{n}$ for all $n \in \mathbb{N}$. We choose $\left(\mu_{n}\right)$ such that

$$
\mu_{n+m}=-i \omega_{n+m}+\left(\sigma_{n}-i \omega_{n+m}\right) / 2 \quad \forall n \in \mathbb{N}
$$


and such that the whole sequence is properly spaced in a vertical strip contained in the open left half-plane. Let $\mathcal{G}$ be the diagonal operator such that $A^{\mu}=-A+\mathcal{G}$. By (3.13) the sequence $\left(f_{n}\right)$ defined below is a Riesz basis in $X$ :

$$
f_{n}=\left[\bar{\mu}_{n} I+\mathcal{G}-A\right]^{-1} B b_{n} .
$$

If we consider $\left(\psi_{n}\right)$ as in $(2.10)$, then under some conditions on $\left(\sigma_{n}\right)$ and $\left(i \omega_{n}\right),\left(f_{n}\right)$ and $\left(\psi_{n}\right)$ will be quadratically close. This is the idea behind Theorem 2.11.

Proof of Theorem 2.11. The proof is similar to that of Theorem 2.9. Let $\left(e_{n}\right)$ be the sequence of eigenvectors of $A$ which is an orthonormal basis in $X$ and let $b_{n}=B^{*} e_{n}$. By exact controllability $\delta=\inf _{n \neq k}\left|\omega_{n}-\omega_{k}\right|>0$. By (2.13) the sequence $\left(\sigma_{n}\right)$ is properly spaced as well as $\left(\mu_{n+m}\right)$. (The proof is elementary but tedious.) We add to $\left(\mu_{n+m}\right) m$ complex numbers $\mu_{1}, \ldots, \mu_{m}$ of negative real part such that $\left(\mu_{k}\right)$ is properly spaced. The sequence $\left(\psi_{n}\right)$ given by (3.10) is a linearly independent sequence of eigenvectors of $A^{K}$. By Theorem 3.8 and Remark 3.9 the sequence $\left(f_{n}\right)$ (as defined in (3.15)) is a Riesz basis in $X$. As $A$ and $A^{\mu}$ commute, the following identity is easy to prove:

$$
\psi_{n}-f_{n+m}=\mathcal{F}_{n}\left(\bar{\mu}_{n+m} I+A^{\mu}\right)^{-1} B b_{n+m},
$$

where

$$
\mathcal{F}_{n}=\left[\left(\bar{\mu}_{n+m}-\sigma_{n}\right) I+\mathcal{G}\right]\left(\sigma_{n} I-A\right)^{-1} .
$$

Note that $\sup _{n \in \mathbb{N}}\left\|\left(\bar{\mu}_{n+m} I+A^{\mu}\right)^{-1} B b_{n+m}\right\|_{X}<\infty$. It is sufficient to prove that for some $\widetilde{n}>m$,

$$
\sum_{n>\widetilde{n}}\left\|\mathcal{F}_{n}\right\|_{\mathcal{L}(X)}^{2}<\infty
$$

Recall that $\mathcal{G}$ is diagonal as defined in Remark 3.9:

$$
\mathcal{G} e_{k}= \begin{cases}\left(\mu_{k}+i \omega_{k}\right) e_{k}, & 1 \leq k \leq m, \\ \left(\sigma_{k-m}-i \omega_{k}\right) e_{k} / 2, & k>m .\end{cases}
$$

The reader can check that (2.12) and (2.13) imply (3.18) (cf. [44]). So Theorem 2.4 and Remark 2.6 can be applied to complete the proof.

\section{Examples based on the one-dimensional wave equation.}

4.1. A 1-D wave equation with boundary feedback. This example is a homogeneous string with tangential force feedback control. The open-loop system is described by

$$
\left\{\begin{array}{l}
w_{t t}(x, t)=w_{x x}(x, t), \quad x \in[0,1], t \geq 0, \\
w(0, t)=0, \\
w_{x}(1, t)=u(t), \quad \text { (input signal) } \\
y(t)=w_{t}(1, t), \quad \text { (output signal) }
\end{array}\right.
$$


where $w(x, t)$ denotes the displacement of the point $x$ of the string at time $t$. Define the Hilbert space $H_{L}^{1}[0,1]=\left\{f \in H^{1}[0,1] \mid f(0)=0\right\}$, with the inner product $\langle f, g\rangle=$ $\int_{0}^{1} f_{x}(x) \overline{g_{x}}(x) \mathrm{d} x$. Let $X=H_{L}^{1}[0,1] \times L^{2}[0,1]$ and let $\|(f, g)\|_{X}^{2}=\int_{0}^{1}\left[\left|f_{x}(x)\right|^{2}+\right.$ $\left.|g(x)|^{2}\right] \mathrm{d} x$. It is not difficult to check that (4.1) defines a regular system with input $u$, output $y$ (both scalar) and state space $X$. The open-loop transfer function $\mathbf{G}$ is

$$
\mathbf{G}(s)=\frac{1-e^{-2 s}}{1+e^{-2 s}}
$$

We consider the feedback operator $K=-\kappa$, with $\kappa>0$, which is easily seen to be admissible. The closed-loop generator $A^{K}$ described below is exponentially stable:

$$
\mathcal{D}\left(A^{K}\right)=\left\{\left[f_{1} f_{2}\right]^{\top} \in X \mid f_{1} \in H^{2}[0,1], f_{2} \in H_{L}^{1}[0,1], f_{1 x}(1)=-\kappa f_{2}(1)\right\}
$$

and

$$
A^{K} f=\left[\begin{array}{cc}
0 & I \\
\frac{\partial^{2}}{\partial x^{2}} & 0
\end{array}\right]\left[\begin{array}{l}
f_{1} \\
f_{2}
\end{array}\right] \quad \forall f \in \mathcal{D}\left(A^{K}\right) .
$$

We apply Theorem 2.9 for reproving a result of Rideau [31]: for $\kappa \neq 1, A^{K}$ is diagonal. As a first step, using the notation $\psi(t)=\left[w(\cdot, t) w_{t}(\cdot, t)\right]^{\top}$, we rewrite the open-loop system in operator form:

$$
\left\{\begin{array}{l}
\dot{\psi}(t)=A \psi(t)+B u(t), \\
y(t)=C_{\Lambda} \psi(t)+D u(t),
\end{array}\right.
$$

where $A$ is the skew-adjoint operator defined by

$$
\mathcal{D}(A)=\left\{\left[f_{1} f_{2}\right]^{\top} \in X \mid f_{1} \in H^{2}[0,1], f_{2} \in H_{L}^{1}[0,1], f_{1 x}(1)=0\right\}
$$

and for all $\left[f_{1} f_{2}\right]^{\top} \in \mathcal{D}(A), A\left[f_{1} f_{2}\right]^{\top}=\left[f_{2} f_{1 x x}\right]^{\top}$. In (4.4), $C \in \mathcal{L}\left(X_{1}, \mathbb{C}\right)$ is defined by $C f=f_{2}(1), B=C^{*} \in X_{-1}, C_{\Lambda}$ is the $\Lambda$-extension of $C$ (see (3.7)) and $D=1$ (see (3.6)). We study the two different cases $0<\kappa<1$ and $\kappa>1$, respectively. The following lemma is contained in the thesis of Rideau [31].

Lemma 4.1. The open-loop generator A has compact resolvents,

$$
\sigma(A)=\left\{\lambda_{n}=i(n-1 / 2) \pi \mid n \in \mathbb{N}\right\} \bigcup\left\{\lambda_{-n}=\overline{\lambda_{n}} \mid n \in \mathbb{N}\right\},
$$

the corresponding eigenvectors $\left(e_{ \pm n}\right)$ form an orthonormal basis in $X$ and the closedloop generator $A^{K}$ has for eigenvalues $\sigma\left(A^{K}\right)$ with simple algebraic multiplicity:

$$
\sigma\left(A^{K}\right)=\left\{\sigma_{ \pm n}=-\sigma \pm i(n-1 / 2) \pi\left|\sigma=\frac{1}{2} \ln \right| \frac{1+\kappa}{1-\kappa} \mid, \quad n \in \mathbb{N}\right\} .
$$

We claim that $(A, B)$ is exactly controllable. Note that this is equivalent to $\left(A, B^{*}\right)$ being exactly observable. To prove this, let $u=0$ in (4.1). Multiplying both sides of (4.1) by $x w_{x}(x, t)$ and integrating by parts, we obtain

$$
\left.\int_{0}^{T} w_{t}^{2}(1, t) \mathrm{d} t=\int_{0}^{T} \int_{0}^{1}\left[w_{t}^{2}(x, t)+w_{x}^{2}(x, t)\right)\right] \mathrm{d} x \mathrm{~d} t
$$




$$
+2 \int_{0}^{1}\left[w_{t}(x, T) x w_{x}(x, T)-w_{t}(x, 0) x w_{x}(x, 0)\right] \mathrm{d} x .
$$

Using the conservation of energy and the Cauchy inequality, we get the admissibility of $B$ together with the exact observability of $\left(A, B^{*}\right)$ :

$$
(T-2)\left\|\left[\begin{array}{c}
w(\cdot, 0) \\
w_{t}(\cdot, t)
\end{array}\right]\right\|_{X}^{2} \leq \int_{0}^{T} w_{t}^{2}(1, t) \mathrm{d} t \leq(T+2)\left\|\left[\begin{array}{c}
w(\cdot, 0) \\
w_{t}(\cdot, t)
\end{array}\right]\right\|_{X}^{2} .
$$

From (3.8), the closed-loop generator is $A^{K}=A+B K(1-K)^{-1} C_{\Lambda}$, which was defined equivalently in (4.3). The spectrum of $A^{K}$, given by the zeros of $1-K \mathbf{G}(s)$, is the spectrum of $A$ shifted to the vertical line $\operatorname{Re} \lambda=-\sigma$. Hence, (2.11) is trivially satisfied with $r=\sigma$ and $m=0$. By Theorem 2.9, $A^{K}$ is Riesz spectral. In fact, it is easy to see that the eigenvalues of $A^{K}$ are algebraically simple.

For $\kappa>1$ the same reasoning works by inverting the flow of information in the system, i.e., exchanging the roles of $u(t)$ and $y(t)$ in (4.1). We can see that

$$
\sigma\left(A^{K}\right)=\left\{\sigma_{ \pm n}=-\sigma \pm i n \pi \mid n \in\{0,1,2, \ldots\}\right\} .
$$

The spectrum of the closed-loop system, given by the zeros of $1-K^{-1} \mathbf{G}^{-1}(s)$ is the spectrum of the open-loop system shifted to the vertical line $\operatorname{Re} \lambda=-\sigma$. Hence, (2.11) is trivially satisfied with $r=\sigma$ and $m=0$. Again by Theorem $2.9, A^{K}$ is Riesz spectral. In fact each eigenvalues of $A^{K}$ is again algebraically simple. Thus, for all $\kappa>0$ except $\kappa=1, A^{K}$ is diagonal. For $\kappa=1$ the spectrum of $A^{K}$ is empty (and then $\mathbb{T}_{2}^{K}=0$ ).

4.2. A 1-D wave equation coupled with a boundary integrator. We consider a system governed by a partial differential equation coupled with an ordinary differential equation, with two inputs and two outputs.

The open-loop system $\Sigma$ is described by the 1 -D wave equation which is cascaded with one integrator on the boundary:

$$
\left\{\begin{array}{lr}
w_{t t}(x, t)=w_{x x}(x, t), & x \in[0,1], t \geq 0, \\
w(0, t)=\xi(t), & w_{x}(1, t)=u_{2}(t), \quad \text { (inputs) } \\
\xi_{t}(t)=u_{1}(t), & y_{2}(t)=-w_{t}(1, t), \quad \text { (outputs) } \\
y_{1}(t)=w_{x}(0, t)-w(0, t),
\end{array}\right.
$$

The system has two inputs $u_{1}$ and $u_{2}$ and two outputs $y_{1}$ and $y_{2}$. We consider the closed-loop system obtained by the following output feedback:

$$
\left[\begin{array}{l}
u_{1}(t) \\
u_{2}(t)
\end{array}\right]=K\left[\begin{array}{l}
y_{1}(t) \\
y_{2}(t)
\end{array}\right]+\left[\begin{array}{l}
v_{1}(t) \\
v_{2}(t)
\end{array}\right], K=\left[\begin{array}{cc}
\kappa_{1} & 0 \\
0 & \kappa_{2}
\end{array}\right], \kappa_{i}>0 .
$$

We write $L^{2}, H^{1}, H^{2}$ instead of $L^{2}[0,1], H^{1}(0,1), H^{2}(0,1)$. The state of our system is $\psi=\left[\begin{array}{ll}w & w_{t}\end{array}\right]^{\top}$ and the state space is

$$
X=\left\{\left[z_{1} z_{2} \xi\right]^{\top} \in H^{1} \times L^{2} \times \mathbb{R} \mid z_{1}(0)=\xi\right\},
$$


with the norm

$$
\left\|\left[\begin{array}{lll}
z_{1} & z_{2} & \xi
\end{array}\right]^{\top}\right\|^{2}=\int_{0}^{1}\left[\left|z_{1 x}(x)\right|^{2}+\left|z_{2}(x)\right|^{2}\right] \mathrm{d} x+|\xi|^{2} .
$$

The system $\Sigma$ in (4.5) is regular with $U=Y=\mathbb{R}^{2}$. The matrix $K$ in (4.6) is an admissible feedback operator for $\Sigma$. Let $A^{K}$ denote the closed-loop generator (defined below). We prove that $A^{K}$ is Riesz spectral.

Define the skew-adjoint operator $A: \mathcal{D}(A) \rightarrow X$ such that

$$
\mathcal{D}(A)=\left\{\left[\begin{array}{ll}
z_{1} & z_{2} \\
\xi
\end{array}\right]^{\top} \in X \cap\left(H^{2} \times H^{1} \times \mathbb{R}\right) \mid z_{1 x}(1)=0, z_{2}(0)=0\right\}
$$

and $A\left[\begin{array}{lll}z_{1} & z_{2} & \xi\end{array}\right]^{\top}=\left[\begin{array}{lll}z_{2} & z_{1 x x} & 0\end{array}\right]^{\top}$. Define $C_{1}, C_{2} \in \mathcal{L}\left(X_{1}, \mathbb{R}\right)$ by

$$
C_{1}\left[\begin{array}{l}
z_{1} \\
z_{2} \\
\xi
\end{array}\right]=z_{1 x}(0)-z_{1}(0), \quad C_{2}\left[\begin{array}{l}
z_{1} \\
z_{2} \\
\xi
\end{array}\right]=-z_{2}(1)
$$

and set $C=\left[\begin{array}{l}C_{1} \\ C_{2}\end{array}\right]$. By direct computation we can find that $B=\left[B_{1} B_{2}\right] \in \mathcal{L}\left(\mathbb{R}^{2}, X_{-1}\right)$ and $B=-C^{*}$. The system (4.5) can be written as

$$
\left\{\begin{array}{l}
\dot{\psi}(t)=A \psi(t)+B u(t), \\
y(t)=C_{\Lambda} \psi(t)+D u(t),
\end{array}\right.
$$

where $D=-I$. The transfer function of the system (4.5) is given by

$$
\mathbf{G}(s)=\left[\begin{array}{cc}
\frac{e^{-s}-e^{s}}{e^{-s}+e^{s}}-\frac{1}{s} & \frac{2}{e^{-s}+e^{s}} \\
\frac{-2}{e^{-s}+e^{s}} & \frac{e^{-s}-e^{s}}{e^{-s}+e^{s}}
\end{array}\right]
$$

and the system is regular.

Lemma 4.2. For $s \in \rho(A),(s I-A)^{-1}$ is compact,

$$
\sigma(A)=\left\{\lambda_{ \pm n}= \pm i\left(n-\frac{1}{2}\right) \pi \mid n \in \mathbb{N}\right\} \bigcup\left\{\lambda_{0}=0\right\}
$$

and the corresponding eigenvectors $\left(e_{ \pm n}\right)$ form an orthonormal basis of $X$ :

$$
e_{k}(x)=\left[\begin{array}{c}
\frac{\sin \left(k-\frac{1}{2}\right) \pi x}{i\left(k-\frac{1}{2}\right) \pi} \\
\sin \left(k-\frac{1}{2}\right) \pi x \\
0
\end{array}\right], \quad e_{-k}(x)=\overline{e_{k}(x)}, \quad e_{0}(x)=\left[\begin{array}{l}
1 \\
0 \\
1
\end{array}\right] .
$$

Remark 4.3. It is easy to check that $C e_{0}=\left[\begin{array}{r}-1 \\ 0\end{array}\right]$ and $C e_{ \pm k}=\left[\begin{array}{c}\mp i \\ (-1)^{k}\end{array}\right]$, $\forall k \in \mathbb{N}$. By Proposition 3.4, $(A, C)$ is exactly observable as well as $\left(A, C_{1}\right)$. However 
$\left(A, C_{2}\right)$ is not. By duality, similar results are true for the exact controllability of $\left(A, B_{1}\right)$ and $\left(A, B_{2}\right)$.

The operators $A^{K}$ and $C^{K}$ are given in (3.8). It is easy to see that

$$
\begin{gathered}
\mathcal{D}\left(A^{K}\right)=\left\{\left[\begin{array}{ll}
z_{1} z_{2} \xi
\end{array}\right]^{\top} \in\left(H^{2} \times H^{1} \times \mathbb{R}\right) \cap X \mid \begin{array}{c}
z_{1 x}(1)=-\kappa_{2} z_{2}(1) \\
z_{2}(0)=\kappa_{1}\left(z_{1 x}(0)-\xi\right)
\end{array}\right\}, \\
A^{K}\left[\begin{array}{l}
z_{1} \\
z_{2} \\
\xi
\end{array}\right]=\left[\begin{array}{c}
z_{2} \\
z_{1 x x} \\
\kappa_{1}\left(z_{1 x}(0)-\xi\right)
\end{array}\right] \quad \forall\left[\begin{array}{l}
z_{1} \\
z_{2} \\
\xi
\end{array}\right] \in \mathcal{D}\left(A^{K}\right) .
\end{gathered}
$$

The following lemmas can be proved by direct computation using Theorem 1.1.

LEMMA 4.4. A number $\lambda \neq 0$ is in $\sigma\left(A^{K}\right)$ if and only if it satisfies

$$
e^{2 \lambda}=\left(\frac{\kappa_{2}-1}{\kappa_{2}+1}\right)\left[\frac{\kappa_{1}+\left(1-\kappa_{1}\right) \lambda}{\kappa_{1}+\left(1+\kappa_{1}\right) \lambda}\right] \text {. }
$$

By $\eta_{n}=\mathcal{O}\left(\xi_{n}\right)$ is meant that $\left|\eta_{n}\right|<\widetilde{C}\left|\xi_{n}\right|, \forall n \in \mathbb{N}$, for some constant $\widetilde{C}>0$. Without loss of generality we study only the case when $\kappa_{1}, \kappa_{2} \in(0,1)$.

Lemma 4.5. Assume that $\kappa_{1}, \kappa_{2} \in(0,1)$. Then there exists some integer $m \geq 0$ such that $\sigma_{k} \in \sigma_{p}\left(A^{K}\right)$ for all $k \in \mathbb{N}$, where

$$
\begin{gathered}
\sigma_{k}=-\beta \pm i \alpha_{k+m}+\mathcal{O}\left(\frac{1}{\alpha_{k+m}}\right), \\
\alpha_{k}=\left(k-\frac{1}{2}\right) \pi, \quad \beta=\ln \sqrt{\frac{\left(1+\kappa_{1}\right)\left(1+\kappa_{2}\right)}{\left(1-\kappa_{1}\right)\left(1-\kappa_{2}\right)}} .
\end{gathered}
$$

Moreover, the eigenvector $\phi_{n}$ of $A^{K}$ corresponding to $\sigma_{n}$ is

$$
\phi_{n}(x)=\left[\begin{array}{c}
f_{n}(x) \\
\sigma_{n} f_{n}(x) \\
f_{n}(0)
\end{array}\right]
$$

where

$$
f_{n}(x)=\frac{1}{i \sigma_{n}}\left\{e^{\sigma_{n} x}+\left[\frac{\left(\kappa_{1}-1\right) \sigma_{n}-\kappa_{1}}{\left(\kappa_{1}+1\right) \sigma_{n}+\kappa_{1}}\right] e^{-\sigma_{n} x}\right\} .
$$

TheOREM 4.6. The closed-loop generator $A^{K}$ is Riesz spectral.

Proof. The sequence $\left(\sigma_{k}\right)$ is easily computed using Rouché's theorem for some large $m$. Recall that $A^{K}=A+B K(I+K)^{-1} C_{\Lambda}$. From (4.10) and using Lemma 4.4 we get easily:

$$
C_{\Lambda} \phi_{n}=\mathcal{S} B^{*} e_{n+m}+\mathcal{O}\left(\frac{1}{\alpha_{n+m}}\right)\left[\begin{array}{l}
1 \\
1
\end{array}\right]
$$


where

$$
\mathcal{S}=\left[\begin{array}{cc}
\frac{2}{\kappa_{1}+1} & 0 \\
0 & \frac{2\left(1-\kappa_{1}\right) e^{\beta}}{\left(1+\kappa_{1}\right)\left(1+\kappa_{2}\right)}
\end{array}\right]
$$

It follows that

$$
\phi_{n}=\left(\sigma_{n}-A\right)^{-1} B K(I+K)^{-1}\left\{\mathcal{S} B^{*} e_{n+m}+\mathcal{O}\left(\frac{1}{\alpha_{n+m}}\right)\left[\begin{array}{l}
1 \\
1
\end{array}\right]\right\} .
$$

The matrix $\widetilde{K}=K(I+K)^{-1} \mathcal{S}$ being diagonal and positive definite, the pair $\left(A, B \widetilde{K}^{\frac{1}{2}}\right)$ is exactly controllable. Applying Corollary 2.3 to $\left(A, B \widetilde{K}^{\frac{1}{2}}\right)$ proves that the following sequence $\left(\widetilde{\phi}_{n}\right)$ is a Riesz basis in $X$ :

$$
\widetilde{\phi}_{n}=-\left[\left(-\beta+i \alpha_{n}\right) I-A\right]^{-1} B \widetilde{K} B^{*} e_{n} .
$$

An argument similar to the proof of Theorem 2.9 tells us that the first sequence in (4.11) is quadratically close to $\left(\widetilde{\phi}_{n+m}\right)$. The second one is quadratically close to zero, because the sequence $\left(\left\|\left(\sigma_{n}-A\right)^{-1} B\right\|_{\mathcal{L}(U, X)}\right)$ is bounded. Hence there exists some integer $m \geq 0$ such that

$$
\sum_{n \in \mathbb{N}}\left\|\widetilde{\phi}_{n+m}-\phi_{n}\right\|_{X}^{2}<\infty
$$

By Theorem 2.7, $A^{K}$ has $m$ other generalized eigenvectors which, concatenated with $\left(\phi_{n}\right)$ like in (2.8), form a Riesz basis in $X$.

Remark 4.7. If $\kappa_{2}=0$ then $A^{K}$ is Riesz spectral using Theorem 2.9 with $r=\beta$.

4.3. Homogeneous string with variable viscous damping. This example has an infinite-rank control operator. We consider the homogeneous string with variable viscous damping:

$$
\left\{\begin{array}{l}
w_{t t}(x, t)-w_{x x}(x, t)+2 a(x) w_{t}(x, t)=0, \quad x \in[0,1], t \geq 0, \\
w(0, t)=w(1, t)=0
\end{array}\right.
$$

where $a \in H^{1}(0,1)$. The eigenvalues and eigenvectors of this system have been studied in detail by Cox and Zuazua [6] in the case $a(x) \geq 0$ for all $x \in[0,1]$ and $a_{0}=\int_{0}^{1} a(x) \mathrm{d} x>0$. One of the open questions in [6] is whether some sequence of generalized eigenvectors of (4.13) forms a Riesz basis in the state space. They needed this Riesz basis property to determine an $a(\cdot)$ resulting in the optimal decay rate of the solutions (see Rao [24] for another result in this direction). Here, using our Theorem 2.4 we prove that the closed-loop generator for (4.13) has a sequence of generalized eigenvectors which is a Riesz basis in the state space.

Consider $H_{0}^{1}(0,1)=\left\{f \in H^{1}(0,1) \mid f(0)=f(1)=0\right\}$. The natural state space for $(4.13)$ is $X=H_{0}^{1}(0,1) \times L^{2}[0,1]$ with $\|f\|_{X}^{2}=\int_{0}^{1}\left[f_{1 x}^{2}+f_{2}^{2}\right] \mathrm{d} x$. Setting $\psi(t)=$ 
$\left[\psi_{1}(t) \psi_{2}(t)\right]^{\top}=\left[w(\cdot, t) w_{t}(\cdot, t)\right]^{\top}$, we associate to (4.13) the following controlled and observed open-loop system:

$$
\left\{\begin{array}{l}
\dot{\psi}(t)=A \psi(t)+B u(t), \quad \psi(0)=\psi_{0} \\
y(t)=C \psi(t)
\end{array}\right.
$$

Here, $A$ is the skew-adjoint operator with compact resolvents given by

$$
\mathcal{D}(A)=\left\{\left[f_{1} f_{2}\right]^{\top} \mid f_{1} \in H^{2}(0,1) \cap H_{0}^{1}(0,1), f_{2} \in H_{0}^{1}(0,1)\right\}
$$

and for all $f=\left[\begin{array}{ll}f_{1} & f_{2}\end{array}\right]^{\top} \in \mathcal{D}(A), A f=\left[\begin{array}{ll}f_{2} & f_{1 x x}\end{array}\right]^{\top}$. The operator $B$ is linear and bounded from $U=L^{2}[0,1]$ to $X$, it has infinite rank and it is given by

$$
B \mathrm{v}(x)=\left[\begin{array}{c}
0 \\
\mathrm{v}(x)
\end{array}\right]
$$

The observation operator $C$ is also linear and bounded from $X$ to $Y=L^{2}[0,1]$ such that $C\left[\begin{array}{ll}f_{1} & f_{2}\end{array}\right]^{\top}(x)=2 a(x) f_{2}(x)$. Thus, $(A, B, C)$ is regular with $D=0$ and every bounded feedback operator $K$ is admissible. The closed-loop system (4.13) is obtained from the open-loop system (4.14) via $u(t)=K y(t)$ with $K=-I$, and $A^{K}=A-B C$. The following result is easy to prove.

Proposition 4.8. The open-loop generator $A$ has the spectrum $\sigma(A)=\sigma_{p}(A)$,

$$
\sigma_{p}(A)=\left\{\begin{array}{l}
\lambda_{n}=i \omega_{n} \\
\lambda_{-n}=-i \omega_{n}
\end{array} \mid \omega_{n}=n \pi, \forall n \in \mathbb{N}\right\}
$$

and the corresponding eigenvectors $\left(e_{n}\right)$ form an orthonormal basis in $X$ :

$$
e_{n}(x)=\left[\begin{array}{l}
\frac{1}{n \pi} \\
i
\end{array}\right] \frac{\widetilde{e}_{n}(x)}{\sqrt{2}}, \quad e_{-n}(x)=\left[\begin{array}{c}
\frac{1}{n \pi} \\
-i
\end{array}\right] \frac{\widetilde{e}_{n}(x)}{\sqrt{2}}, \quad n \in \mathbb{N},
$$

where $\widetilde{e}_{n}(x)=\sqrt{2} \sin (n \pi x), n \in \mathbb{N}$, form an orthonormal basis in $L^{2}[0,1]$.

Note that $B^{*} e_{n}(x)=i \sin \pi n x$ and $B^{*} e_{-n}(x)=-B^{*} e_{n}(x)$ for all $n \in \mathbb{N}$. Since $\left(i \omega_{ \pm n}\right)$ is properly spaced, $(A, B)$ is exactly controllable because $\left(\left\|B^{*} e_{ \pm n}\right\|_{U}\right)$ is bounded from below by a positive number (see [32]). Similarly, we have that $C e_{ \pm n}(x)= \pm i \sqrt{2} a(x) \widetilde{e}_{n} .(A, C)$ is exactly observable if and only if on some interval $[a, b] \subset[0,1]$ with $b-a>0, \inf _{x \in[a, b]}|a(x)|>0$. Using Fourier series in (4.14), we can show that this is equivalent to the existence of a $\widetilde{L}>0$ such that

$$
\int_{0}^{2}\|C \psi(t)\|_{L^{2}[a, b]}^{2} \mathrm{~d} t \geq \widetilde{L}\left\|\psi_{0}\right\|_{X}^{2} \quad \forall \psi_{0} \in X .
$$

In (4.13) the feedback operator is infinite-rank and depends on the space variable $x \in[0,1]$. To apply Bari's theorem, we have to construct a Riesz basis with which we compare a sequence of generalized eigenvectors. However, this Riesz basis is not 
so easy to obtain as in the case $U=\mathbb{C}$. Therefore, some asymptotic estimates of eigenvalues and eigenvectors of the closed-loop system are necessary.

It is easy to see that $\lambda \in \sigma\left(A^{K}\right)$ if and only if the following equation has a non-trivial solution:

$$
\left\{\begin{array}{l}
\lambda^{2} w(x)-w_{x x}(x)+2 \lambda a(x) w(x)=0 \\
w(0)=w(1)=0
\end{array}\right.
$$

We set

$$
a_{0}=\int_{0}^{1} a(\xi) \mathrm{d} \xi, \quad \xi(x)=\int_{0}^{x} a(\xi) \mathrm{d} \xi-a_{0} x .
$$

As $m$ is sufficiently large, the closed-loop system has an eigenvalue in each of the disjoint disks $D_{n}$ centered at $-a_{0}+i(n+m) \pi, n \in \mathbb{N}$, with radius small enough to make them disjoint. We denote by $\sigma_{n}$ the eigenvalue in $D_{n}$ and by $\sigma_{-n}$ the eigenvalue in $D_{-n}$. Let $w_{n}(s)$ denote the non-trivial solution of (4.15) corresponding to $\lambda=\sigma_{n}$. The eigenvector of $A^{K}$ corresponding to $\sigma_{n}$ is written as

$$
\phi_{n}(x)=\left[\begin{array}{l}
w_{n}(x) \\
\sigma_{n} w_{n}(x)
\end{array}\right] .
$$

The following result has been obtained in [6].

Proposition 4.9. There exist some positive integer $m$ and some $K>0$ such that the following estimates hold for all $n \in \mathbb{N}$ :

$$
\left\{\begin{array}{l}
\left|\sigma_{ \pm n}-\left(-a_{0} \pm i(n+m) \pi\right)\right| \leq \frac{K}{(n+m)}, \\
\left|w_{ \pm n x}(x)-\cosh \left(\sigma_{ \pm n} x+\int_{0}^{x} a(\xi) \mathrm{d} \xi\right)\right| \leq \frac{K}{\left|\sigma_{ \pm n}\right|}, \\
\left|\sigma_{ \pm n} w_{ \pm n}(x)-\sinh \left(\sigma_{ \pm n} x+\int_{0}^{x} a(\xi) \mathrm{d} \xi\right)\right| \leq \frac{K}{\left|\sigma_{ \pm n}\right|} .
\end{array}\right.
$$

Using Proposition 4.9 and our Theorem 2.4 we show that some sequence of generalized eigenvectors of $A^{K}$ forms a Riesz basis in $X$.

TheOREm 4.10. With the notation of Proposition 4.9, there exists a positive integer $m$ such that $A^{K}$ has $m$ generalized eigenvectors, denoted by $\widetilde{\psi}_{1}, \widetilde{\psi}_{2}, \ldots, \widetilde{\psi}_{m}$, so that the sequence $\left(\widetilde{\psi}_{1}, \widetilde{\psi}_{2}, \ldots, \widetilde{\psi}_{m}, \phi_{1}, \phi_{2}, \ldots\right)$ is a Riesz basis in $X$. Here, $\phi_{k}$ is the eigenvector of $A^{K}$ defined in (4.17).

Proof. Denote $L_{0}^{2}[0,1]=\left\{f \in L^{2}[0,1] \mid \int_{0}^{1} f(x) \mathrm{d} x=0\right\}$. Put $\widetilde{X}=L_{0}^{2}[0,1] \times$ $L^{2}[0,1]$, with the usual inner product. Let $\Lambda_{1}: X \rightarrow \widetilde{X}$ be defined by $\Lambda_{1}[f g]^{\top}=$ $\left[\begin{array}{ll}f_{x} & g\end{array}\right]^{\top}$. Then $\Lambda_{1}$ is an isometric isomorphism. It transforms the orthonormal basis $\left(e_{ \pm n}\right)$ in $X$ into the following orthonormal basis in $\widetilde{X}$ :

$$
\left(\varpi_{ \pm n}\right)=[\cos (n \pi x), \pm i \sin (n \pi x)]^{\top}, \quad n \in \mathbb{N} .
$$

Define $\Lambda_{2}: \widetilde{X} \rightarrow \widetilde{X}$ such that for all $f=\left[f_{1} f_{2}\right]^{\top} \in \tilde{X}$,

$$
\left(\Lambda_{2} f\right)(x)=\left[\begin{array}{cc}
\cosh \xi(x) & \sinh \xi(x) \\
\sinh \xi(x) & \cosh \xi(x)
\end{array}\right] f(x)-\left\langle f,\left[\begin{array}{c}
\cosh \xi(\cdot) \\
\sinh \xi(\cdot)
\end{array}\right]\right\rangle_{L^{2} \times L^{2}} \cdot\left[\begin{array}{l}
1 \\
0
\end{array}\right]
$$


with $\xi(x)$ defined as in (4.16). We claim that $\Lambda_{2}$ is continuous and invertible from $\widetilde{X}$ onto $\widetilde{X}$. Indeed, it is not difficult to check that $\Lambda_{2}$ is bounded, one-to-one and onto. Hence, the sequence $\left(\psi_{n}\right)=\left(\Lambda_{2} \varpi_{n}\right)$ is a Riesz basis in $\widetilde{X}$. Integrating by parts we see that the following two sequences are both in $l^{2}$ :

$$
\int_{0}^{1} \cosh (\xi(x)) \cos (n \pi x) \mathrm{d} x, \quad \int_{0}^{1} \sinh (\xi(x)) \sin (n \pi x) \mathrm{d} x .
$$

Note also that $\|f\|_{X}=\left\|\Lambda_{1} f\right\|_{\tilde{X}}$. By these facts and the estimates in Proposition 4.9, there exists some integer $m>0$ such that

$$
\sum_{n \in \mathbb{N}}\left\|\phi_{ \pm n}-\Lambda_{1}^{-1} \psi_{ \pm(n+m)}\right\|_{X}^{2}<+\infty
$$

Now applying Theorem 2.4 finishes the proof.

In Cox and Zuazua [6, Section 6], Theorem 4.10 is proved by a different method, with the less restrictive assumption that $a(x)$ has bounded variation. However, they impose $a(x) \geq 0$ (which we do not assume) and one step in their proof is unclear (applying Rouché's theorem on an unbounded domain).

\section{Examples based on the beam equation.}

5.1. An Euler-Bernoulli beam with boundary force feedback. Consider the beam equation with boundary shear force feedback control:

$$
\left\{\begin{array}{l}
w_{t t}(x, t)+w_{x x x x}(x, t)=0 \\
w(0, t)=w_{x}(0, t)=w_{x x}(1, t)=0, \\
w_{x x x}(1, t)=\kappa w_{t}(1, t), \quad \kappa>0 .
\end{array}\right.
$$

We prove that a sequence of generalized eigenvectors of the semigroup generator associated to the system (5.1) forms a Riesz basis in the state space. This answers an open question raised by Chen et al in [4] and studied by Conrad and Morgul [5]. Our approach gives a complete positive answer to this question.

The associated open-loop system $\Sigma$ with input $u$ and output $y$ is described by

$$
\left\{\begin{array}{l}
w_{t t}(x, t)+w_{x x x x}(x, t)=0 \\
w(0, t)=w_{x}(0, t)=w_{x x}(1, t)=0 \\
w_{x x x}(1, t)=-u(t) \\
y(t)=w_{t}(1, t)
\end{array}\right.
$$

The state of $\Sigma$ is $\left[\begin{array}{ll}w & w_{t}\end{array}\right]^{\top}$ and the state space is the separable Hilbert space

$$
X=\left\{[f g]^{\top} \in H^{2}(0,1) \times L^{2}[0,1] \mid f(0)=f_{x}(0)=0\right\}
$$

with the norm $\left\|[f g]^{\top}\right\|_{X}^{2}=\int_{0}^{1}\left[\left|f_{x x}(x)\right|^{2}+|g(x)|^{2}\right] \mathrm{d} x$. With the notation $x(t)=$ $\left[w(\cdot, t) w_{t}(\cdot, t)\right]^{\top}$ we write $(5.2)$ as follows:

$$
\left\{\begin{array}{l}
\dot{x}(t)=A x(t)+B u(t) \\
y(t)=C_{\Lambda} x(t)
\end{array}\right.
$$


where $A$ is the skew-adjoint operator with compact resolvents defined by

$$
\mathcal{D}(A)=\left\{\begin{array}{l|l}
{[f g]^{\top} \in X \cap\left(H^{4}(0,1) \times H^{2}(0,1)\right)} & \begin{array}{l}
g(0)=g_{x}(0)=0, \\
f_{x x}(1)=f_{x x x}(1)=0
\end{array}
\end{array}\right\}
$$

and for all $[f g]^{\top}$ in the domain, $A[f g]^{\top}=\left[g-f_{x x x x}\right]^{\top}, C[f g]^{\top}=g(1)$ and $B=C^{*}$.

The formulation (5.3) is possible because $\Sigma$ is regular, see Rebarber [26]. We know from [27, Theorem 2.7] that every $K=-\kappa$, with $\kappa>0$ is an admissible feedback operator for $\Sigma$. The closed-loop generator corresponding to (5.1) is $A^{K}=A+B K B_{\Lambda}^{*}$ with a suitable domain (see [42]). Notice that $\left(A, B^{*}\right)$ is exactly observable. Indeed, multiplying $(5.2)$ (with $u(t)=0$ ) by $x w_{x}(x, t)$ and integrating, we obtain

$$
\begin{aligned}
& \int_{0}^{T} w_{t}^{2}(1, t) \mathrm{d} t=\int_{0}^{T} \int_{0}^{1}\left[w_{t}^{2}(x, t)+3 w_{x x}^{2}(x, t)\right] \mathrm{d} x \mathrm{~d} t \\
& \quad+2 \int_{0}^{1}\left[w_{t}(x, T) x w_{x}(x, T)-w_{t}(x, 0) x w_{x}(x, 0)\right] \mathrm{d} x .
\end{aligned}
$$

Since $\|x(t)\|_{X}$ is constant, there exist positive constants $k_{1}, k_{2}$ and $T_{0}$ such that

$$
k_{1}\left\|\left(w(\cdot, 0), w_{t}(\cdot, 0)\right)\right\|_{X}^{2} \leq \int_{0}^{T_{0}} w_{t}^{2}(1, t) \mathrm{d} t \leq k_{2}\left\|\left(w(\cdot, 0), w_{t}(\cdot, 0)\right)\right\|_{X}^{2} .
$$

Hence, $(A, B)$ is exactly controllable.

TheOrem 5.1. For all $\kappa>0$, the generator $A^{K}$ for (5.1) is Riesz spectral.

Proof. $\lambda \in \sigma_{p}(A)$ if and only if $\lambda=i \omega^{2}$ (or $-i \omega^{2}$ ) and $\omega>0$ satisfying

$$
f(\omega)=\cos \omega+\frac{1}{\cosh \omega}=0 .
$$

A number $\sigma \in \sigma_{p}\left(A^{K}\right)$ if and only if $\sigma=i \tau^{2}$ (or $-i \bar{\tau}^{2}$ ) and the complex number $\tau \neq 0$ satisfies $f(\tau)-g(\tau)=0$, where

$$
g(\tau)=\frac{i \kappa}{\tau}[\tanh \tau \cos \tau-\sin \tau] .
$$

Setting $\alpha_{k}=k \pi+\frac{\pi}{2}$ and $r_{k}=\frac{1+\kappa}{\alpha_{k}}$ we consider the set of open disks $D_{k}=D\left(\alpha_{k}, r_{k}\right)$, $k \in \mathbb{N}$ (centered at $\alpha_{k}$ with radius $r_{k}$ ). Both $f(z)$ and $f(z)-g(z)$ are analytic in each disk $D_{k}$ and

$$
\lim _{k \rightarrow+\infty} \sup _{z \in \partial D_{k}}\left|\frac{f(z)}{g(z)}\right|=\frac{1+\kappa}{\kappa}>1 .
$$

For $k$ sufficiently large, by Rouché's theorem, $f(z)-g(z)$ has one and only one zero in each disk $D_{k}$, just as $f(z)$. Let $\left(\lambda_{n}\right)$ be the sequence in $\mathbb{C}$ such that $\sigma_{p}(A)=\left\{\lambda_{ \pm n}=\right.$ $\left.\pm i \omega_{n} \mid n \in \mathbb{N}\right\}$. Thus there exist an integer $m>0$ and a constant $K_{1}>0$ such that

$$
\left|\omega_{n}-\alpha_{n}^{2}\right| \leq \frac{K_{1}}{\alpha_{n}} \quad \forall n>m .
$$


Set $\beta_{n}=-2 \kappa+i \alpha_{n+m}^{2}$. Using Rouché's theorem we prove that for some integer $m>0$, there exists a sequence $\left(\sigma_{n}\right)$ such that $\Lambda=\left\{\sigma_{n} \mid n \in \mathbb{N}\right\} \cup\left\{\bar{\sigma}_{n} \mid n \in \mathbb{N}\right\}$ is a subset of $\sigma_{p}\left(A^{K}\right)$ and such that $\left|\sigma_{n}-\beta_{n}\right| \leq \frac{K_{2}}{\alpha_{n+m}}$ for some constant $K_{2}>0$ and all $n \in \mathbb{N}$. Clearly $\sigma_{k} \neq \sigma_{l} \forall k \neq l$. Each $\sigma_{n}, n \in \mathbb{N}$ is algebraically simple for some $m>0$ (see Conrad and Morgul [5]), but we do not need this fact. It is easy to see that $\sum_{n \in \mathbb{N}}\left|-2 \kappa+i \omega_{n+m}-\sigma_{n}\right|^{2}<\infty$. By Theorem 2.9, $A^{K}$ is Riesz spectral.

5.2. Coupled beams with a dissipative joint. Consider the following Euler-Bernoulli beam equation with the stabilizing feedback $u(t)=-\kappa w_{t}(d, t)+v(t)$ acting at the junction point $d \in(0,1)$. The open-loop system is described by

$$
\left\{\begin{array}{l}
w_{t t}(x, t)+w_{x x x x}(x, t)=0, \quad x \in(0, d) \cup(d, 1), \\
w(0, t)=w_{x x}(0, t)=0, w_{x}(1, t)=w_{x x x}(1, t)=0, \\
w\left(d^{+}, t\right)=w\left(d^{-}, t\right), w_{x}\left(d^{+}, t\right)=w_{x}\left(d^{-}, t\right), \\
w_{x x}\left(d^{+}, t\right)=w_{x x}\left(d^{-}, t\right), \\
w_{x x x}\left(d^{+}, t\right)-w_{x x x}\left(d^{-}, t\right)=u(t) .
\end{array}\right.
$$

Here, $u$ is the input signal and $\kappa>0$ is a constant. We consider also the output function $y(t)=w_{t}(d, t)$.

A necessary and sufficient condition for the exponential stability of the closed-loop system corresponding to (5.7) has been proved in Rebarber [27], using a frequency domain approach. In [27] Rebarber asked whether the spectrum-determined growth condition is satisfied for this closed-loop system. Here, we show that a set of generalized eigenvectors of this closed-loop system form a Riesz basis in the state space if $d$ belongs to a certain (dense) subset $\mathcal{R}$ of $(0,1)$. This $\mathcal{R}$ (which is defined in the next lemma) is the same set for which the systems (open and closed loop) are exactly controllable and the closed-loop system is exponentially stable. It follows that the spectrum-determined growth condition is satisfied for the closed-loop system when $d \in \mathcal{R}$. This gives a positive answer to a question asked in [27].

We consider the system (5.7) in the state space $X=H_{L R}^{2}(0,1) \times L^{2}[0,1]$, where $H_{L R}^{2}(0,1)=\left\{f \in H^{2}[0,1] \mid f(0)=f_{x}(1)=0\right\}$. With $\psi(t)=\left[\psi_{1}(t) \psi_{2}(t)\right]^{\top}=$ $\left[w(\cdot, t) w_{t}(\cdot, t)\right]^{\top}$, the open-loop system associated with $(5.7)$ is written as

$$
\left\{\begin{array}{l}
\dot{\psi}(t)=A \psi(t)+B u(t), \\
y(t)=B_{\Lambda}^{*} \psi(t),
\end{array}\right.
$$

where

$$
\begin{gathered}
\mathcal{D}(A)=\left\{\left[\begin{array}{l}
f \\
g
\end{array}\right] \in\left(H^{4}(0,1) \times H_{L R}^{2}(0,1)\right) \cap X \mid f_{x x}(0)=f_{x x x}(1)=0\right\}, \\
A\left[\begin{array}{l}
f \\
g
\end{array}\right]=\left[\begin{array}{cc}
0 & I \\
-\partial_{x}^{4} & 0
\end{array}\right] \quad \forall\left[\begin{array}{l}
f \\
g
\end{array}\right] \in \mathcal{D}(A),
\end{gathered}
$$


and $B^{*}[f g]^{\top}=g(d)$. Recall that $A$ is the generator of a unitary group on $X$, the triple $(A, B, C)$ is regular and every $K \in \mathbb{R}$ is an admissible feedback operator for $\Sigma$ (see [27, Lemma 2.6]).

Lemma 5.2. Let $A$ and $B$ be defined as above. Then

$$
\sigma(A)=\sigma_{p}(A)=\left\{\lambda_{ \pm n}=i \omega_{ \pm n} \mid \omega_{ \pm n}= \pm(n-1 / 2)^{2} \pi^{2}, \quad n \in \mathbb{N}\right\}
$$

and $(A, B)$ is exactly controllable if and only if $d \in \mathcal{R}$, where $\mathcal{R}$ is the set of rational numbers in $(0,1)$ with coprime factorization $d=\frac{p}{q}$, with $p$ odd.

Proof. Note that $A$ has compact resolvents. Direct computation proves (5.9) and the corresponding eigenvectors are given by

$$
\Phi_{ \pm n}=\frac{1}{\sqrt{2}}\left[\begin{array}{cc}
\frac{1}{\lambda_{ \pm n}} & 1
\end{array}\right]^{\top} \phi_{n}
$$

where $\phi_{n}(x)=\sin \left(\omega_{n} x\right)$. The sequence $\left(\Phi_{ \pm n}\right)$ forms an orthonormal basis in $X$ and $\left|B^{*} \Phi_{n}\right|=|\sin ((n-1 / 2) \pi d)|$. From Ingham [15], $\left(A, B^{*}\right)$ is exactly observable (or, $(A, B)$ is exactly controllable) if and only if there is some constant $c>0$ such that

$$
|\sin ((n-1 / 2) \pi d)| \geq c \quad \forall n \in \mathbb{N} .
$$

This is true if and only if $d=p / q$ with odd $p$ (see [27, Lemma 2.9]).

Now we take the Laplace transform in (5.7) with respect to time. Using Mathematica the transfer function $\mathbf{G}(s)$ is computed with $s=i \tau^{2}$ :

$$
\mathbf{G}\left(i \tau^{2}\right)=\frac{i}{2 \tau}\left[\frac{\cos (d-1) \tau \sin d \tau \cosh \tau-\cos \tau \cosh (d-1) \tau \sinh d \tau}{\cosh \tau \cos \tau}\right] .
$$

The closed-loop system corresponding to (5.8) with the feedback $K=-\kappa$, i.e., $u=$ $-\kappa y+v$, is governed by

$$
\dot{\psi}(t)=A \psi(t)+B K B_{\Lambda}^{*} \psi(t)+B v=A^{K} \psi(t)+B v,
$$

where

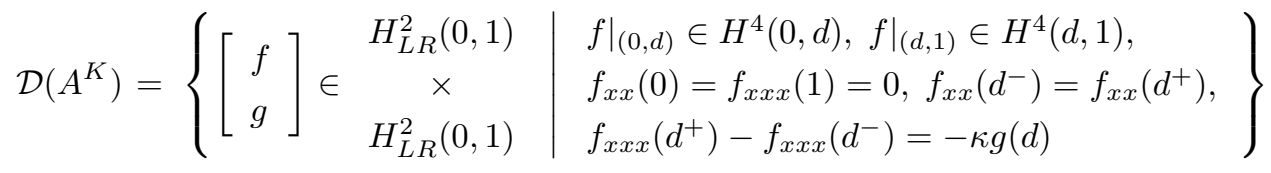

and

$$
A^{K}[f g]^{\top}=\left[g-f_{x x x x}\right]^{\top}
$$

From Theorems 1.1, the eigenvalues of $A^{K}$ are the solutions of

$$
1-K \mathbf{G}(s)=0 .
$$


The solutions are of the form $s=i \tau^{2}$, where

$$
\begin{gathered}
\frac{i \kappa}{2 \tau}[\cos (d-1) \tau \sin d \tau \cosh \tau-\cos \tau \cosh (d-1) \tau \sinh d \tau] \\
+\cosh \tau \cos \tau=0 .
\end{gathered}
$$

TheOREm 5.3. If $d \in \mathcal{R}$, then $A^{K}$ has a set of generalized eigenvectors which forms a Riesz basis in $X$. Moreover, the eigenvalues of $A^{K}$ are algebraically simple, possibly with the exception of a finite number of them.

Proof. It is sufficient to prove (2.13) in Theorem 2.11 because (2.12) is evident. Let $m \in \mathbb{N}$ and let the numbers $\alpha_{n}(n \in \mathbb{N})$ be defined by

$$
\alpha_{n}=i \omega_{(n+m)}-\kappa \sin ^{2}(n+m-1 / 2) d \pi,
$$

where $i \omega_{n+m} \in \sigma_{p}(A) \forall n \in \mathbb{N}$. Using Rouché's theorem we can prove that for some large integer $m>0$, there exists a sequence $\left(\sigma_{n}\right)$ such that $\sigma_{n}$ and $\bar{\sigma}_{n}$ are eigenvalues of $A^{K}$ and such that $\left|\alpha_{n}-\sigma_{n}\right| \leq \widetilde{K} /(n+m)$ for some constant $\widetilde{K}>0$ (see also Guo and Chan [10]). It is obvious that $\sigma_{l} \neq \sigma_{k}$ for $l \neq k$ when $m$ is large enough. Hence (2.13) is satisfied. Thus the proof is complete.

5.3. Coupled beams with two dissipative controls. The system consists of coupled beams with both a force and a torque feedback at the coupling joint. With the simultaneous feedback of force and torque present in action the system is always exponentially stabilized as proved in Tucsnak and Weiss [38] using the Huang-Prüss theorem. This prevents the well-known lack of robustness of exponential stabilization by force feedback or by torque feedback alone (cf. Rebarber [27] and see also Subsection 5.2). In this subsection we prove that the exponentially stabilized system is Riesz spectral. As a consequence of our result not only is the system always exponentially stabilized but also may its decay rate be assigned arbitrarily by means of a simultaneous feedback.

The system of coulped beams controlled by both force control and torque control is described by the following PDE:

$$
\begin{aligned}
& W_{t t}+W_{x x x x}=0, \quad x \in(0,1) \backslash\{\xi\}, \\
& W(0, t)=W(1, t)=0, W_{x x}(0, t)=W_{x x}(1, t)=0,[W]_{\xi}=\left[W_{x}\right]_{\xi}=0,
\end{aligned}
$$

where $\xi$ is some constant belonging to $(0,1)$ and $[W]_{\xi}$ denotes the jump of $W$ at $\xi:[W]_{\xi}=W\left(\xi^{+}, t\right)-W\left(\xi^{-}, t\right)$. The boundary condition for $(5.13)$ is complemented by the controls

$$
\left[\left[W_{x x x}\right]_{\xi} W_{x t}(\xi, t)\right]^{\top}=\left[\begin{array}{ll}
u_{1} & u_{2}
\end{array}\right]^{\top} .
$$


The output measure that we consider is as follows

$$
y(t)=\left[W_{t}(\xi, t)-\left[W_{x x}\right]_{\xi}\right]^{\top} .
$$

The exponentially stabilizing output feedback law is given by $u=K y$ where

$$
K=-\left[\begin{array}{cc}
\kappa_{1} & 0 \\
0 & \frac{1}{\kappa_{2}}
\end{array}\right], \quad \kappa_{1}, \kappa_{2}>0 .
$$

On computing formally the time derivative of the total mechanic energy $E(t)=$ $\int_{0}^{1}\left(W_{t}^{2}+W_{x x}^{2}\right) d x$ we observe that the energy is decreasing in time for the closed-loop system:

$$
\dot{E}(t)=-\kappa_{1}\left[W_{t}(\xi, t)\right]^{2}-\frac{1}{\kappa_{2}}\left(\left[W_{x x}\right]_{\xi}\right)^{2} \leq 0 .
$$

Let $H_{0}^{2}(0,1)=\left\{f \in H^{2}(0,1) \mid f(0)=f(1)=0\right\}$ and let $X=H_{0}^{2}(0,1) \times L^{2}(0,1)$ with the inner product $\left\langle f, g>_{X}=\int_{0}^{1}\left(f_{1 x x} g_{1 x x}+f_{2} g_{2}\right) d x\right.$. The closed-loop system is exponentially stable for any $\xi \in(0,1)$ (see Tucsnak and Weiss [38]). The main result that we will prove in this subsection is concerned with the case $\xi=1 / 2$. The general case is an open question to the best of our knowledge.

Theorem 5.4. Assume that $\kappa_{1}, \kappa_{2}>0$ and $\xi=1 / 2$. Then the generator $A^{K}$ of the closed-loop system (5.13)-(5.16) is Riesz spectral in the state space $X$.

From now on we set $\xi=1 / 2$. Define the unbounded operator $A$ by

$$
\mathcal{D}(A)=\left\{\begin{array}{l|l}
f=\left[\begin{array}{l}
f_{1} \\
f_{2}
\end{array}\right] \in X & \begin{array}{l}
\chi_{[0, \xi]} f_{1} \in H^{4}(0, \xi), \chi_{[\xi, 1]} f_{1} \in H^{4}(\xi, 1) \\
f_{2} \in H_{0}^{2}(0,1), f_{1 x x}(0)=f_{1 x x}(1)=0 \\
{\left[f_{1 x x x}\right]_{\xi}=0, f_{2 x}(\xi)=0}
\end{array}
\end{array}\right\},
$$

and for all $f \in \mathcal{D}(A), A f=\left[f_{2}-f_{1 x x x x}\right]^{\top}$. It is easy to see that $A$ is skew-adjoint and diagonal. It is the generator of a $C_{0}$ semigroup on $X$ and has compact resolvents. Define the observation operator $C: X_{1} \rightarrow \mathbb{R}^{2}$ such that

$$
C f=\left[C_{1} f C_{2} f\right]^{\top}=\left[f_{2}(\xi)-\left[f_{1 x x}\right]_{\xi}\right]^{\top} .
$$

The control operator $B: \mathbb{R}^{2} \rightarrow X_{-1}$ is collocated: $B=C^{*}$. As proved in Rebarber [27] $(A, B, C)$ is a regular triple with the feedthrough operator $D=0$ and $K$ in (5.16) is admissible. Hence the closed-loop generator $A^{K}=A+C^{*} K C_{\Lambda}$ is described by

$$
\mathcal{D}\left(A^{K}\right)=\left\{\begin{array}{l|l}
f=\left[\begin{array}{l}
f_{1} \\
f_{2}
\end{array}\right] \in X & \begin{array}{l}
\chi_{[0, \xi]} f_{1} \in H^{4}(0, \xi), \chi_{[\xi, 1]} f_{1} \in H^{4}(\xi, 1) \\
f_{2} \in H_{0}^{2}(0,1), f_{1 x x}(0)=f_{1 x x}(1)=0 \\
{\left[f_{1 x x x}\right]_{\xi}=-\kappa_{1} f_{2}(\xi), f_{2 x}(\xi)=\left(1 / \kappa_{2}\right)\left[f_{1 x x}\right]_{\xi}}
\end{array}
\end{array}\right\},
$$


and for all $f \in \mathcal{D}\left(A^{K}\right), A^{K} f=\left[f_{2}-f_{1 x x x x}\right]^{\top}$. As $A^{K}=A$ for $\kappa_{1}=1 / \kappa_{2}=0$ their eigenvalues and eigenvectors are computed on the same time.

Indeed $\lambda \in \sigma_{p}\left(A^{K}\right)$ if and only if $\lambda=i \omega^{2}$ with $\operatorname{Re} \omega \geq 0$ and the following differential equation has a non-trivial solution:

$(5.20)[\varphi]_{\xi}=\left[\varphi_{x}\right]_{\xi}=0, \varphi_{x}(\xi)=\left(\frac{1}{\kappa_{2} i \omega^{2}}\right)\left[\varphi_{x x}\right]_{\xi},\left[\varphi_{x x x}\right]_{\xi}=-\kappa_{1} i \omega^{2} \varphi(\xi)$.

Moreover, if we approximately normalize a such $\varphi$ in $L^{2}[0,1]$ such that $0<|\varphi|_{L^{2}}<\infty$ then the corresponding eigenvector of $A^{K}$ is given by

$$
\phi=\left[\begin{array}{c}
\frac{\varphi}{i \omega^{2}} \\
\varphi
\end{array}\right] \text {. }
$$

We compute directly eigenvalues and eigenvectors for $A^{K}$ because those of $A$ can be obtained by taking $K=0$.

Lemma 5.5. For some integer $\widetilde{m}>0$ the two sequences $\left(i \omega_{2 n}^{2}\right)$ and $\left(i \omega_{2 n+1}^{2}\right)$ are in $\sigma_{p}\left(A^{K}\right)$, where

$$
\begin{gathered}
\omega_{2 n}=2(n+\widetilde{m}) \pi+\frac{\pi}{2}+\frac{i 2}{(n+\widetilde{m}) \pi \kappa_{2}}+\mathcal{O}\left(\frac{1}{(n+\widetilde{m})^{2}}\right) \quad \forall n \in \mathbb{N}, \\
\omega_{2 n+1}=(2(n+\widetilde{m})+1) \pi+\frac{i \kappa_{1}}{4(n+\widetilde{m}) \pi}+\mathcal{O}\left(\frac{1}{(n+\widetilde{m})^{2}}\right) \quad \forall n \in \mathbb{N} .
\end{gathered}
$$

Moreover the corresponding eigenvectors are given via (5.21) by

$$
\varphi_{2 n}(x)= \begin{cases}\frac{\sinh \omega_{2 n} x}{\cosh \left(\omega_{2 n} / 2\right)}-\left[1+\mathcal{O}\left(\frac{1}{\omega_{2 n}}\right)\right] \frac{\sin \omega_{2 n} x}{\cos \left(\omega_{2 n} / 2\right)}, & x \in(0,1 / 2), \\ \frac{\sinh \omega_{2 n}(x-1)}{\cosh \left(\omega_{2 n} / 2\right)}-\left[1+\mathcal{O}\left(\frac{1}{\omega_{2 n}}\right)\right] \frac{\sin \omega_{2 n}(x-1)}{\cos \left(\omega_{2 n} / 2\right)}, & x \in(1 / 2,1),\end{cases}
$$

$$
\varphi_{2 n+1}(x)= \begin{cases}\mathcal{O}\left(\frac{1}{\omega_{2 n+1}}\right) \frac{\sinh \omega_{2 n+1} x}{\cosh \left(\omega_{2 n+1} / 2\right)}-\left[1+\mathcal{O}\left(\frac{1}{\omega_{2 n+1}}\right)\right] \sin \omega_{2 n+1} x, & x \in(0,1 / 2), \\ \mathcal{O}\left(\frac{1}{\omega_{2 n+1}}\right) \frac{\sinh \omega_{2 n+1}(x-1)}{\cosh \left(\omega_{2 n+1} / 2\right)}+\sin \omega_{2 n+1}(x-1), & x \in(1 / 2,1) .\end{cases}
$$

Proof. General solution of (5.18)-(5.20) is given by

$$
\varphi(x)= \begin{cases}c_{1} \sinh \omega x+c_{2} \sin \omega x, & x \in(0,1 / 2), \\ c_{3} \sinh \omega(x-1)+c_{4} \sin \omega(x-1), & x \in(1 / 2,1),\end{cases}
$$

where the constants $c_{l}, l=1, \ldots, 4$ satisfy the following linear algebraic equations:

$$
c_{1} \sinh \omega \xi+c_{2} \sin \omega \xi-c_{3} \sinh \omega(\xi-1)-c_{4} \sin \omega(\xi-1)=0,
$$




$$
\begin{array}{r}
c_{1}\left(\cosh \omega \xi-\frac{i \sinh \omega \xi}{\omega \kappa_{2}}\right)+c_{2}\left(\cos \omega \xi+\frac{i \sin \omega \xi}{\omega \kappa_{2}}\right)+\frac{c_{3} i \sinh \omega(\xi-1)}{\omega \kappa_{2}} \\
-\frac{c_{4} i \sin \omega(\xi-1)}{\omega \kappa_{2}}=0,
\end{array}
$$$$
\frac{-c_{1} i \sinh \omega \xi}{\omega \kappa_{2}}+\frac{c_{2} i \sin \omega \xi}{\omega \kappa_{2}}+c_{3}\left(\cosh \omega(\xi-1)+\frac{i \sinh \omega(\xi-1)}{\omega \kappa_{2}}\right)
$$

$$
+c_{4}\left(\cos \omega(\xi-1)-\frac{i \sin \omega(\xi-1)}{\omega \kappa_{2}}\right)=0
$$

$$
c_{1}\left(\cosh \omega \xi-\frac{i \kappa_{1} \sinh \omega \xi}{\omega}\right)-c_{2}\left(\cos \omega \xi+\frac{i \kappa_{1} \sin \omega \xi}{\omega}\right)-c_{3} \cosh \omega(\xi-1)
$$

$$
+c_{4} \cos \omega(\xi-1)=0 .
$$

The solution (5.26) is non-trivial if and only if the determinant of the coefficient matrix is zero. The determinant is equal to the following:

$$
\begin{array}{r}
\operatorname{det}(\omega)=\left\{\sinh \omega \xi \cos \omega \xi-\sin \omega \xi \cosh \omega \xi+\frac{4 i \sinh \omega \xi \sin \omega \xi}{\omega \kappa_{2}}\right\} \\
\cdot\left\{4 \cosh \omega \xi \cos \omega \xi+\frac{i \kappa_{1}(\cosh \omega \xi \sin \omega \xi-\cos \omega \xi \sinh \omega \xi)}{\omega}\right\}
\end{array}
$$

Using Rouché's theorem we find zeros of $\operatorname{det}(\omega)$ of large module, as estimated in (5.22) and (5.23).

When $\omega=\omega_{2 n}$, the equations (5.28)-(5.30) are linearly independent for some $\widetilde{m}$ sufficiently large, and the solutions form a one-dimensional linear space:

$$
\begin{aligned}
& c_{2}=\left[-1+\mathcal{O}\left(1 / \omega_{2 n}\right)\right] \sec \omega_{2 n} \xi \cosh \omega_{2 n} \xi c_{1}, \quad c_{3}=\left[1+\mathcal{O}\left(1 / \omega_{2 n}\right)\right] c_{1}, \\
& c_{4}=\left[-1+\mathcal{O}\left(1 / \omega_{2 n}\right)\right] \sec \omega_{2 n} \xi \cosh \omega_{2 n} \xi c_{1} .
\end{aligned}
$$

Taking $c_{1}=1 / \cosh \omega_{2 n} \xi$ we get (5.24).

Let $\omega=\omega_{2 n+1}$ and $c_{4}=1$. First we solve (5.27) for $c_{2}$ and then sustitute it into (5.28) and (5.29) to obtain $c_{1}$ and $c_{3}$. This proves (5.25).

Proof of Theorem 5.4. The reader proves easily that $A$ is skew-adjoint and has compact resolvents. A purely imaginary number $\lambda$ is an eigenvalue of $A$ if and only if $\lambda=i \omega^{2}$ where $\omega \geq 0$ satisfies (5.31) for $\kappa_{1}=1 / \kappa_{2}=0$. We denote the sequences in (5.22) and (5.23) by $\left(\widetilde{\omega}_{2 n}\right)$ and $\left(\widetilde{\omega}_{2 n+1}\right)$ when $\kappa_{1}=1 / \kappa_{2}=0$. There exists some integer $\widetilde{m}$ such that all the zeros of $\operatorname{det}(\omega)$ that are larger than $2 \widetilde{m}$ are given by these two sequences. Moreover each eigenvalue $\lambda=i \widetilde{\omega}_{2 n}$ or $i \widetilde{\omega}_{2 n+1}$ is geometrically simple as proved above. Since $\operatorname{det}(\omega)$ is analytic, (5.31) has only a finite number of zeros in 
$[0,2 \widetilde{m}]$, denoted by $z_{1}, \ldots, z_{m}$. We order the eigenvalues of $A$ as well as some of $A^{K}$ as follows:

$$
\lambda_{k}=i z_{k}^{2} \quad \forall 1 \leq k \leq m, \quad \lambda_{2 n-1+m}=i \widetilde{\omega}_{2 n+1}^{2}, \quad \lambda_{2 n+m}=i \widetilde{\omega}_{2 n}^{2} \quad \forall n \in \mathbb{N},
$$

and

$$
\sigma_{2 n-1}=i \omega_{2 n+1}^{2}, \quad \sigma_{2 n}=i \omega_{2 n}^{2}, \quad \forall n \in \mathbb{N} .
$$

We denote by $e_{n+m}$ and $\varphi_{n}$, respectively, their eigenvectors ordered accordingly for $n \in \mathbb{N}$ as defined in (5.22)-(5.23). Using the trigonometric and hyperbolic function relations we prove easily the following:

$$
\left|e_{n+m}(x)-\varphi_{n}(x)\right| \leq \mathcal{O}\left(\frac{1}{n+\widetilde{m}}\right) .
$$

Using (5.21) we check that the condition (2.7) is satisfied. Applying Theorem 2.4 completes the proof of Theorem 5.4.

The pair $(A, C)$ is exactly observable but neither $\left(A, C_{1}\right)$ nor $\left(A, C_{2}\right)$ is. With the same notation as in the proof of Theorem 5.4, let $\phi_{n}$ and $\phi_{n}^{K}$ be the eigenvectors for the open-loop and close-loop systems, respectively:

$$
\phi_{n}=\left[\begin{array}{c}
\frac{e_{n}}{i \widetilde{\omega}_{n}^{2}} \\
e_{n}
\end{array}\right], \quad \phi_{n}^{K}=\left[\begin{array}{c}
\frac{\varphi_{n}}{i \omega_{n}^{2}} \\
\varphi_{n}
\end{array}\right] .
$$

Remark that $A^{K}=A+C^{*} K C_{\Lambda}$ and $\left|C_{\Lambda} \phi_{n}^{K}-C \phi_{n+m}\right|=\mathcal{O}(1 /(n+\widetilde{m}))$. The same reasoning as in the proof of Theorem 4.6 allows us to prove Theorem 5.4. It seems that for the beam examples the sequence of eigenvectors of the closed-loop system is quadratically close to that of the open-loop system. However it is not the case for the string examples.

Acknowledgements. Part of the work has been done while the second author was visiting the University of Lyon in 2005. Part of the work was carried out while the first author was visiting the Institute of Systems Science, Chinese Academy of Sciences, between 1998 and 2009 and he acknowledges the interesting discussions with Dr. Bao-Zhu Guo, Professor in the Chinese Academy of Sciences, about Riesz bases during the visits.

\section{REFERENCES}

[1] S.A. Avdonin and S.A. Ivanov, Families of Exponentials: The Method of Moments in Controllability Problems for Distributed Parameter Systems, Cambridge University Press, 1995.

[2] J. Baillieul and M. Levi, Rotational elastic dynamics, Physica 27D(1987), pp.43-62. 
[3] G. Chen, M.C. Delfour, A.M. Krall, and G. Payre, Modelling, stabilization and control of serially connected beams, SIAM J. Control \& Optim. 25(1987), pp. 526-546.

[4] G. Chen, S.G. Krantz, D.W. Ma, C.E. Wayne, and H.H. West, The Euler-Bernoulli beam equation with boundary energy dissipation, in: Operator Methods for Optimal Control Problems, ed.: S.J. Lee, Marcel Dekker, New York, pp.67-96, 1987.

[5] F. Conrad and O. Morgul, On the stabilization of a flexible beam with a tip mass, SIAM J. Control \& Optim. 36(1998), pp. 1962-1986.

[6] S. Cox And E. Zuazua, The rate at which energy decays in a damped string, Commun. in Partial Differential Equations 19:1\& 2(1994), pp. 213-243.

[7] N. Dunford And J.T. Schwartz, Linear Operators, Part III, Wiley-Interscience, New York, 1971.

[8] I.C. Gohberg And M.G. Kreîn, Introduction to the Theory of Linear Nonselfadjoint Operators, American Math. Society, Providence, Rhode Island, 1969.

[9] B.Z. GuO, Riesz basis approach to the stabilization of a flexible beam with a tip mass, SIAM J. Control \& Optimization 39(2001), pp.1736-1747.

[10] B.Z. Guo AND K.Y. Chan, Riesz basis generation, eigenvalues distribution, and exponential stability for an Euler-Bernoulli beam with joint feedback control, Revista Matemática Complutense 14(2001), pp.1-24.

[11] B.Z. Guo And Y.H. Luo, Riesz basis property of a second order hyperbolic system with collocated scalar input-output, IEEE Trans. Autom. Control 47(2002), pp.693-698.

[12] S. HANSEn ANd G. Weiss, New results on the operator Carleson measure criterion, IMA Journal of Math. Control and Information 14(1997), pp.3-32.

[13] L.F. Ho, Spectral assignability of systems with scalar control and application to a degenerate hyperbolic system, SIAM J. Control \& Optim. 24(1986), pp.1212-1231.

[14] L.F. Ho And D. Russell, Admissible input elements for systems in Hilbert space and a Carleson measure criterion, SIAM J. Control \& Optim. 21(1983), pp.614-640.

[15] A.E. Ingham, Some trigonometric inequalities with applications to the theory of series, Math. Zeitschrift 41(1936), pp.367-379.

[16] B. JACOB AND H. ZWART, Exact observability of diagonal systems with a finite-dimensional output operator, Systems \& Control Letters 43(2001), pp.101-109.

[17] S. Jaffard, M. Tucsnak, And E. Zuazua, On a theorem of Ingham, J. Fourier Anal. and Appl. 3(1997), pp. 577-582.

[18] T. Kato, Perturbation Theory for Linear Operators, Springer-Verlag, Berlin, 1980.

[19] V. Komornik, Exact Controllability and Stabilisation: The Multiplier Method, Masson and John Wiley, Paris and Chichester, 1994.

[20] I. Lasiecka AND TRIGgiani, Finite rank, relatively bounded perturbations of semigroup generators. Pert II: spectrum and Riesz basis assignment with application to feedback systems, Ann. Mat. Pura Appl. 143(1986), pp.47-100.

[21] J.Q. Liu, Perturbation of one rank and pole assignment, J. Systems Science and Mathematical Sciences 2(1982), pp. 81-94 (in Chinese with English abstract).

[22] K. LiU, Z. LIU, AND B. RAO, Exponential stability of an abstract nondissipative linear system, SIAM J. Control \& Optim. 40(2001), pp.149-165.

[23] N.K. Nikol'skil, Treatise on the Shift Operator, Springer-Verlag, New York, 1986.

[24] B.P. RAO, Optimal energy decay rate in a damped Rayleigh beam, Contemporary Mathematics 209 (eds.: S. Cox and I. Lasiecka), pp.221-229, American Mathematical Society, Providence, Rhode Island, 1997.

[25] R. RebARBer, Spectral assignability for distributed parameter systems with unbounded scalar control, SIAM J. Control \& Optim. 26(1988), pp. 1362-1387.

[26] R. ReBARBer, Conditions for the equivalence of internal and external stability for distributed parameter systems, IEEE Trans. Autom. Control 38(1993), pp.995-998. 
[27] R. RebArber, Exponential stability of coupled beams with dissipative joints: a frequency domain approach, SIAM J. Control \& Optim. 33(1995), pp.1-28.

[28] R. Rebarber and S. Townley, Robustness and continuity of the spectrum for certain distributed parameter systems, Automatica 31(1995), pp.1533-1546.

[29] R. Rebarber and G. Weiss, Necessary conditions for exact controllability with a finitedimensional input space, Systems \& Control Letters 40(2000), pp.217-227.

[30] R. Rebarber And H. Zwart, Open-loop stabilizability of infinite-dimensional systems, Math. Control, Signals and Systems 11(1998), pp.129-160.

[31] P. RideAu, Contrôle d'un assemblage de poutres flexibles par des capteurs-actionneurs ponctuels: étude du spectre du système, Thèse, Ecole nationale supérieure des Mines de Paris, Sophia-Antipolis, France, 1985.

[32] D. Russell and G. Weiss, A general necessary condition for exact observability, SIAM J. Control \& Optim. 32(1994), pp.1-23.

[33] D. Salamon, Infinite-dimensional linear systems with unbounded control and observation: a functional analytic approach, Trans. of the American Math. Society 300(1987), pp.383431.

[34] G. Sklyar and A. Rezounenko, A theorem on the strong asymptotic stability and determination of stabilizing controls, C.R. Acad. Sci. Paris 333(2001), pp.807-812.

[35] O. Staffans and G. Weiss, Transfer functions of regular linear systems. Part II: the system operator and the Lax-Phillips semigroup, Trans. of the American Math. Society 354(2002), pp. 3229-3262.

[36] O. Staffans and G. Weiss, Transfer functions of regular linear systems. Part III: inversions and duality, Integral Equations and Operator Theory 49(2004), pp.517-558.

[37] S.H. Sun, On spectrum distribution of completely controllable linear systems, SIAM J. Control \& Optim. 19(1981), pp.730-743.

[38] M. TUCSNAK And G. Weiss, How to get a conservative well-posed linear system out of thin air, Part I: Well-posedness and energy balance, ESAIM Control Optim. Calc. Var. 9(2003), pp. 247-274.

[39] M. Tucsnak And G. Weiss, Observation and Control for Operator Semigroups, BirkhäuserVerlag, Basel, 2009.

[40] G. WEIss, Admissible observation operators for linear semigroups, Israel J. Math. 65(1989), pp. 17-43.

[41] G. Weiss, Transfer functions of regular linear systems, Part I: Characterizations of regularity, Trans. Amer. Math. Society 342(1994), pp. 827-854.

[42] G. Weiss, Regular linear systems with feedback, Mathematics of Control, Signals and Systems 7(1994), pp. 23-57.

[43] G. Weiss And C.Z. Xu, Spectral properties of infinite-dimensional closed-loop systems, Mathematics of Control, Signals and Systems 17(2005), pp.153-172.

[44] C.Z. Xu And G. Sallet, On spectrum and Riesz basis assignment of infinite dimensional linear systems by bounded linear feedbacks, SIAM J. Control \& Optimization 34(1996), pp. 521-541.

[45] C.Z. Xu AND J. BAIllieul, Stabilizability and stabilization of a rotating body-beam system with torque control, IEEE Trans. Autom. Contr. 38(1993), pp. 1754-1765. 